\title{
Layered Wireless Video Relying on Minimum-Distortion Inter-Layer FEC Coding
}

\author{
Yongkai Huo, Mohammed El-Hajjar, Rob G. Maunder and Lajos Hanzo, Fellow, IEEE \\ School of ECS, University of Southampton, UK. \\ Email: \{yh3g09, meh, rm, lh\}@ecs.soton.ac.uk, http://www-mobile.ecs.soton.ac.uk
}

\begin{abstract}
Layered video coding is capable of progressively refining the reconstructed video quality with the aid of multiple layers of unequal importance. When the base layer $(B L)$ is corrupted or lost due to channel impairments, the enhancement layers (ELs) must be discarded by the video decoder, regardless whether they are perfectly decoded or not, which implies that the transmission power assigned to the ELs is wasted. To circumvent this problem, we proposed a bit-level inter-layer forward error correction (IL-FEC) scheme for layered video transmission in our previous work, which implanted the systematic bits of the BL into the systematic bits of the ELs using exclusive-OR operations (XOR). This allowed the receiver to exploit the implanted bits of the ELs for assisting the BL's decoding and hence improved the overall system performance of our IL-FEC aided layered video scheme. In this treatise, we find the specific FEC coding rates in a real-time on-line fashion for the sake optimizing the overall system performance. The proposed procedure is widely applicable to diverse wireless transceivers and FEC codecs. Our simulation results show that the proposed optimized IL-FEC system outperforms the traditional optimal UEP by about 1.9 dB of $E_{b} / N_{0}$ at a peak signal-to-noise ratio (PSNR) of $38 \mathrm{~dB}$. Viewing the improvements in terms of the video quality, $3.3 \mathrm{~dB}$ of PSNR improvement is attained at an $E_{b} / N_{0}$ of $10 \mathrm{~dB}$, when employing a recursive systematic convolutional (RSC) code.
\end{abstract}

\section{INTRODUCTION}

In recent years, low-delay lip-synchronized wireless video communications became a reality and the road to this is detailed in [1]. Layered video coding [2] has been widely adopted by the existing video coding standards [3]-[6], which is capable of generating multiple layers of unequal importance. The most important layers are referred to as the base layer (BL), while the less important layers depend on the BL and are referred to as enhancement layers (ELs). As an inherent component of layered video coding standards, partitioned mode video coding [5] encodes each video-frame slice into at most three partitions, namely partition $\mathrm{A}, \mathrm{B}$ and $\mathrm{C}$ according to the error sensitivity of the bistreams, where partition $\mathrm{A}$ may be deemed to be the BL, while partitions $\mathrm{B}$ and $\mathrm{C}$ are treated as ELs. Furthermore, the moving picture expert group (MPEG)'s [1] developed a multiview profile (MVP) [3], where the left view and right view are encoded as a BL and an EL, respectively. Another recent layered video coding standard, referred to as scalable video coding (SVC) [4], [5] was designed as an extension of H.264/AVC [5]. In SVC, a video sequence is encoded into a stream containing multiple

Copyright (c) 2013 IEEE. Personal use of this material is permitted. However, permission to use this material for any other purposes must be obtained from the IEEE by sending a request to pubs-permissions@ieee.org.

The financial support of the EU's Concerto project, of the EPSRC under the auspices of the India-UK Advanced Technology Centre (IU-ATC) and that of the ERC's Advanced Fellow Grant is gratefully acknowledged. dependent layers, where a number of subset layers may be extracted and hence the bitrate may be controlled according to the users' specific requirements in practical video services. A common characteristic of layered video coding scheme is that the ELs may be assigned a lower priority than the BL and hence may be discarded in the scenario of network congestion or buffer overflow [7]. Moreover, when the related $\mathrm{BL}$ is corrupted or lost due to channel impairments, the layered video decoder has to discard the corresponding ELs, regardless whether they are correctly recovered or not.

Unequal error protection (UEP) was first proposed in [8], where a stronger forward error correction (FEC) code was allocated to the more important data and a weaker FEC code was allocated to the less important data. In [9] UEP was achieved by optimizing the transmission power assigned to individual source bits according to their importance. Additionally, UEP using convolutional codes was investigated in [10], where the rate-compatible convolutional codes (RCPC) proposed in [11] were employed. Moreover, a number of UEP techniques based on low-density parity-check (LDPC) codes were studied in [12]-[15], where the more important bits were more strongly protected than the less important bits. Moreover, most of the above-mentioned UEP contributions considered artificial signals, rather than realistic video signals, which may not be directly extended to the scenario of flawless, low-delay, lip-synchronized video communications.

In layered video coded bitstreams different layers have a different importance, which implies that employing UEP techniques would substantially improve the reconstructed video quality. Generally, these UEP techniques [16]-[31] may be classified into two categories ${ }^{1}$, namely the packet-level schemes [18], [20], [23]-[27], [30] and bit-level schemes [16], [17], [19], [21], [22], [28], [29], [31]. Specifically, the packet-level contributions [18], [20], [23]-[27], [30] mitigate the packet loss events as exemplified by the packets lost in Internet-routers [32], while the bit-level ones are devoted to eliminating bit-errors of wireless scenarios [33]. Let us continue by considering the family of packet-level UEP schemes first [18], [20], [25], [26]. An UEP scheme was conceived for object-based video communications in [18] for achieving the best attainable system performance under specific bitrate and delay constraints in an error-prone network environment. A novel UEP method using Reed-Solomon codes was proposed in [20] for SVC video transmission over networks inflicting packet-loss events. Firstly, the layer-weighted expected zone of error propagation (LW-EZEP) was defined as an efficient performance metric for quantifying the error propagation ef-

${ }^{1}$ Thanks to an anonymous reviewer for this valuable suggestion. 
fects imposed by packet loss events. Then, the corresponding Reed-Solomon (RS) coding rates were assigned based on LWEZEP for minimizing the expected video distortion. Crosslayer operation aided scalable video streaming designed for error-prone channels was investigated in [25], where the RS coded UEP was optimized for robust video delivery. The expected video quality was evaluated based on both the available bandwidth and the packet loss ratio (PLR) encountered, which was then further improved by employing content-aware bitrate allocation. Finally, an error concealment method was invoked at the receiver. The authors of [26] studied an UEP scheme using Luby Transform (LT) codes for recovering the video packets lost owing to network congestions. The abovementioned UEP methodologies tended to employ hard decoded FEC [34] codes and dispensed with soft-information exchange in the decoding process, since they are carried out at the application layer. Similarly, bit-level UEP techniques have also been widely investigated [16], [17], [19], [21], [22], [29]. In [17], an UEP scheme using a turbo transceiver was optimized for wireless video telephony. The UEP performance of data-partitioned [5] H.264/AVC video streaming systems using recursive systematic convolutional (RSC) codes was evaluated in [29], while turbo coded modulation based UEP was investigated in [19], where both the cutoff rates and the channel capacity of each of the UEP levels was determined. The authors of [21] considered the unequal importance of both the video-frames in a group of pictures (GOP) and the significance of the diverse macroblocks (MB) in a video frame for transmission over wireless channels, where a prompt and efficient fast rate allocation scheme was also investigated. However, only three protection classes were discussed in [21], which limits the attainable system performance. The same authors also considered the different importance of the intracoded (I) frame and of the predicted $(\mathrm{P})$ frames within a GOP in [22], where the video bits of different importance were mapped to the different-integrity modulation constellation points of hierarchical quadrature amplitude modulation (QAM). These bit-level schemes tended to employ physical layer FEC codes [35] and perform soft decoding [34] for wireless video communications.

In the traditional UEP scheme conceived for layered video communication, variable-rate FEC codes were assigned to the different-sensitivity layers for improving the reconstructed video quality. However, when the BL is corrupted or lost, the ELs have to be discarded by the video decoder, regardless whether they are perfectly decoded or not, which implies that both the transmission power and the bandwidth assigned to the ELs is wasted. Hence it is beneficial improve the protection of the more important BL with the aid of the ELs. Hence, the authors of [24] proposed a packet-level solution based on fountain codes [23] for scalable video multicast over networks inflicting packet loss events, where ELs conveyed parity information protecting the more important BL. By contrast, hierarchical network coding [27] and generalized network coding [30] techniques were also proposed for combating packet-loss events, when transmitting information of unequal importance. Furthermore, a bit-level layer-aware FEC (LA-FEC) philosophy using a hard-decoded Raptor code was designed for scalable video transmission over the binary erasure channels (BEC) in [28], [36]. The Raptor encoder generated the parity bits right across the BL and the ELs at the transmitter. As a benefit, the parity bits of the ELs may be utilized for assisting in correcting the errors residing in the BL at the receiver. Motivated by these advances, we developed a bit-level inter-layer coded FEC (IL-FEC) scheme layered video telephony over wireless fading channels relying on a soft-decoded systematic FEC code in [31], where the systematic bits of the BL are implanted into the ELs at the transmitter. At the receiver, the BL's bits implanted into the ELs may be utilized for correcting the BL. The abovementioned IL-FEC technique of [31] was also combined with the UEP philosophy for the sake of further improving the attainable system performance. In [31], a number of coding rates were tested for the sake of proving the benefits of the proposed IL technique, where the code rates arrangements were determined empirically. However, in practical scenarios, different configurations of video codecs and different video sequences may have different characteristics, which may require different channel coding rates for achieving the best system performance.

Against this background, in this treatise, we propose a technique for finding the optimized coding rates for coded bitstreams "on-the-fly" at the transmitter, which optimizes the IL-FEC coded system performance. Specifically, we find the coding rates achieving the minimum video quality distortion with the aid of the mutual information (MI) between the loglikelihood ratios (LLRs) and the corresponding video bits. In this context, the soft-decoding metric of the FEC codec and of the demodulator are characterized by lookup tables (LUTs), since these cannot be characterized theoretically. Finally, the widely employed SVC-H.264 codec will be utilized for the sake of benchmarking our proposed system. In a nutshell, we focus our attention on the optimization of bit-level IL-FEC encoded scalable video communications over wireless fading channels. Specifically, our proposed technique is significantly different from the existing contributions [16], [20], [21], [23], [25], [26], [28] in the following aspects. Firstly, our technique is proposed for layered video communication over wireless channels, while the packet-level contributions of [20], [23], [25], [26] consider networks, which inflict packet-loss events or on networks modeled by the BEC. Secondly, IL-FEC relies on bit-level soft-decoding aided channel decoders, such as an RSC code, while [20], [23], [25], [26], [28] considered hard-decoded Reed-Solomon or fountain codes. Moreover, we propose a solution employing near-continuous coding rates, while only three protection rates - namely low-order, mediumorder and high-order protection - were utilized in [16], [21], which limits the system's performance. Finally, we find the optimized coding rates for the IL-FEC aided UEP system of [31], where the basic principle of IL-FEC was conceived, but the assisted focus and the system optimization was set aside for further research. Against this background, the rationale and novelty of this paper is summarized as follows.

1) We set out to optimize soft-decoding bit-level IL-FEC encoded scalable video communication over wireless 
fading channels relying on a new objective function (OF) for the sake of finding the best code rate. Specifically, our technique relies on the MI and LUTs for finding the optimized coding rates in a real-time, online fashion, while minimizing video quality distortion. In contrast to the packet-level contributions of [20], [23], [25], [26], where the authors considered hard-decoded video streams transmitted over networks, which inflict packetloss events or networks modelled by BEC, we consider transmission over non-dispersive Rayleigh fading wireless channels, where the layered video stream is protected by FEC codes and then soft-decoded at the receiver.

2) We utilize the MI and LUTs for the sake of optimizing the proposed system's performance, where we show an $E_{b} / N_{0}$ gain of $1.9 \mathrm{~dB}$ at a PSNR of $38 \mathrm{~dB}$. Alternatively, viewing the improvements in terms of the attainable video quality, or a PSNR gain of $3.3 d B$ is achieved at an $E_{b} / N_{0}$ of $10 \mathrm{~dB}$ over the benchmarker of [21].

3) A general solution is proposed for optimizing the coding rate allocation for arbitrary non-iterative FEC codes protecting, arbitrary layered video codecs. More explicitly, our solution is applicable to arbitrary transceivers provided that the receiver is capable of generating nearGaussian distributed soft-information.

We use the SVC-H.264/AVC scheme in our simulations, but our proposed scheme is not limited to SVC, it can be readily applied in any arbitrary system relying on layered video coding, such as the partitioned video coding regime of [31]. The rest of this paper is organized as follows. Section II details our proposed IL-FEC system model and the related video transmission techniques. Then the coding-rate optimization of the system is detailed in Section III. The performance of our optimized IL-FEC scheme using a RSC codec is benchmarked in Section IV using two video sequences having different motion characteristics. Finally, we offer our conclusions in Section V.

\section{System OVERVIEW}

In this section, we briefly introduce the architecture of the inter-layer FEC scheme conceived for scalable video transmission, while further details on this technique may be found in [31]. The architecture of the system is displayed in Fig. 1, where the SVC mode of H.264 [5] is employed. Moreover, two layers, namely the BL $L_{0}$ and the EL $L_{1}$ are assumed to be generated by the SVC encoder, noting that more complex scenarios may be readily applied - we simply use two layers here for ease of explanation ${ }^{2}$. The variable node decoder (VND) and check node decoder (CND) [37] used in Fig. 1 are illustrated in Fig. 2. Both the VND and CND blocks accept a number of soft information inputs and generate the related soft information outputs for iteratively exploiting the deliberately imposed inter-dependencies between the coded layers $L_{0}$ and $L_{1}$. Specifically, assuming that $u_{1}, u_{2}$ and $u_{3}=u_{1} \oplus u_{2}$ are random binary variables, the VND of Fig. 2 sums two LLR

${ }^{2}$ In our results in Section IV, we show how our proposed techniques work for SVC encoder using three layers.

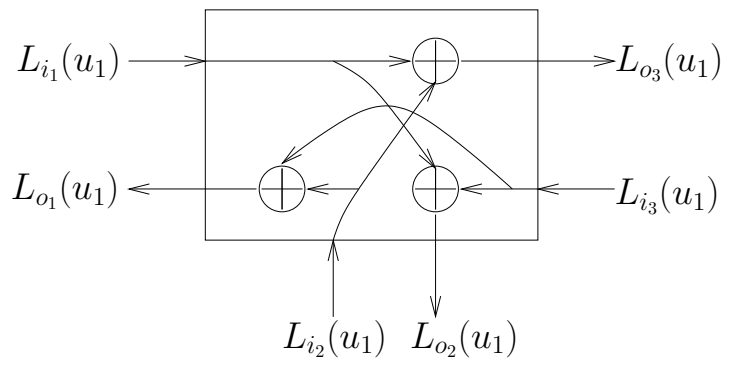

(a) VND

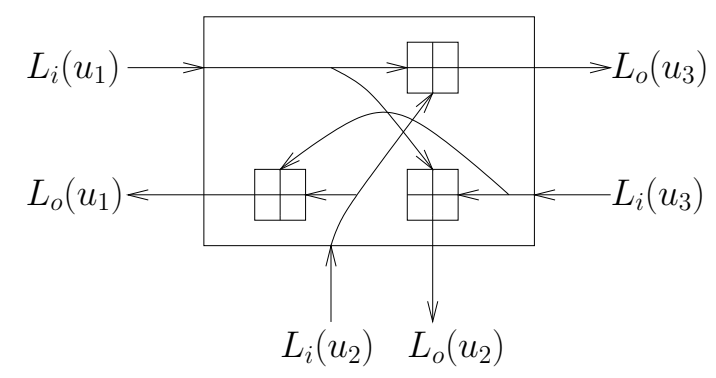

(b) CND

Fig. 2: The structure of VND and CND, where $\oplus$ and $\boxplus$ represent the addition and boxplus action, respectively. $L_{i}(\cdot)$ and $L_{o}(\cdot)$ represent the input and output LLR, respectively.

inputs for generating an improved LLR output, which may be formulated as $L_{o_{3}}\left(u_{1}\right)=L_{i_{1}}\left(u_{1}\right)+L_{i_{2}}\left(u_{1}\right)$. Given the confidence of the bits $u_{1}$ and $u_{2}$, the boxplus operation of $L\left(u_{3}=u_{1} \oplus u_{2}\right)=L\left(u_{1}\right) \boxplus L\left(u_{2}\right)$ [38] may be utilized for deriving the confidence of the bit $u_{3}$. Specifically, the boxplus operation $\boxplus$ may be formulated as follows [39]

$$
\begin{aligned}
& L\left(u_{1}\right) \boxplus L\left(u_{2}\right)=\log \frac{1+e^{L\left(u_{1}\right)} e^{L\left(u_{2}\right)}}{e^{L\left(u_{1}\right)}+e^{L\left(u_{2}\right)}} \\
& =\operatorname{sign}\left[L\left(u_{1}\right)\right] \cdot \operatorname{sign}\left[L\left(u_{2}\right)\right] \cdot \min \left[\left|L\left(u_{1}\right)\right|,\left|L\left(u_{2}\right)\right|\right] \\
& +\log \left[1+e^{-\left|L\left(u_{1}\right)+L\left(u_{2}\right)\right|}\right]-\log \left[1+e^{-\left|L\left(u_{1}\right)-L\left(u_{2}\right)\right|}\right] .
\end{aligned}
$$

In contrast to the VND block, the CND operation of Fig. 2 may be expressed as $L_{o}\left(u_{3}\right)=L_{i}\left(u_{1}\right) \boxplus L_{i}\left(u_{2}\right)$ for calculating the LLR of bit $u_{3}$, given the LLR input of the bits $u_{1}$ and $u_{2}$.

In Section II-A, the IL-FEC encoding techniques invoked at the transmitter are illustrated followed by our IL-FEC decoding techniques in Section II-B, where we emphasize on how the VND and the CND can exploit the IL information for improving the overall system performance. In Section IIA and II-B, we assume that the layers $L_{0}$ and $L_{1}$ contain an identical number of bits for simplifying our explanations, but our algorithm may be readily applied to the scenario of having an unequal number of bits as in [31].

\section{A. Transmitter Model}

At the transmitter of Fig. 1, the video source signal $s$ is compressed using the SVC of the H.264 encoder, generating layers $L_{0}$ and $L_{1}$. Then the output bitstream $x$ is de-multiplexed into the bitstreams of $x_{0}$ and $x_{1}$ by the DEMUX block of Fig. 1, where $x_{0}$ and $x_{1}$ represent the bitstream of the BL $L_{0}$ and of the EL $L_{1}$, respectively. Afterwards, the resultant two layers 


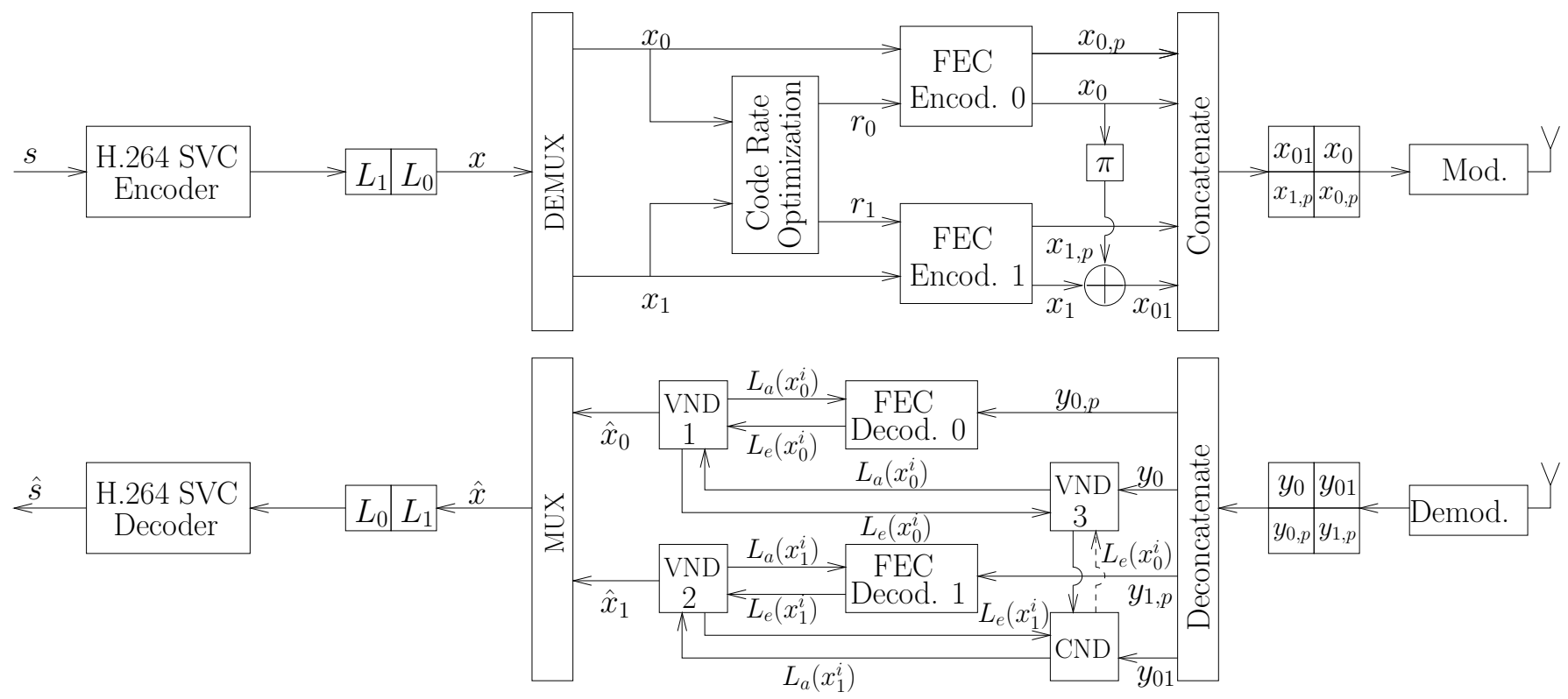

Fig. 1: Block diagram of the proposed IL-FEC coded SVC H.264/AVC coded video system, where $r_{0}$ and $r_{1}$ represent the code rates for FEC encoder 0 and 1, respectively. The "Code Rate Optimization" block will be detailed in Section III.

are encoded as follows:

1) The BL bit sequence $x_{0}$ representing $L_{0}$ will be encoded by the FEC encoder 0 of Fig. 1, which results in the encoded bits containing the systematic bits $x_{0}$ and parity bits $x_{0, p}$.

2) The bit sequence of the EL $x_{1}$ representing $L_{1}$ will firstly be encoded into the systematic bits $x_{1}$ and the parity bits $x_{1, p}$ by the FEC encoder 1 . Then the XOR operation will be utilized for implanting the systematic information of $x_{0}$ into the systematic information of $x_{1}$, which is carried out without changing the parity bits $x_{1, p}$ of the EL $L_{1}$, as shown in Fig. 1. Specifically, the implantation process results in the check bits of $x_{01}^{i}=x_{0}^{i} \oplus x_{1}^{i}$. After this procedure, both the check bits $x_{01}^{i}$ and the parity bits $x_{1, p}$ are output.

Finally, the bit sequences $x_{0}, x_{0, p}, x_{01}$ and $x_{1, p}$ are concatenated into a joint bitstream for transmission. Note however that the layers $x_{0}$ and $x_{1}$ may contain a different number of bits. Additionally, the interleavers $\pi_{1}$ are employed for interleaving the BL $x_{0}$, before its XOR-based implantation into the EL $x_{1}$. Following the IL-FEC encoding procedure, the resultant bits are modulated by the binary phase-shift keying (BPSK) modulator of Fig. 1 and then transmitted over non-dispersive uncorrelated Rayleigh fading wireless channels.

\section{B. Receiver Model}

In this section, we exemplify the IL decoding process using the BL $L_{0}$ and the EL $L_{1}$. At the receiver ${ }^{3}$, BPSK demodulation is performed, which generates the LLRs containing the systematic information $y_{0}, y_{01}$ and the parity information $y_{0, p}$ and $y_{1, p}$, for the layers $L_{0}$ and $L_{1}$, respectively. Following

\footnotetext{
${ }^{3}$ The deinterleavers $\pi^{-1}$ is not shown in the receiver of Fig. 1 for the sake of simplifying the system architecture.
}

the demodulator, the IL-FEC decoder of Fig. 1 is invoked for exchanging extrinsic information across the two layers. Firstly, the FEC decoder 0 will decode the received information $y_{0}$ and $y_{0, p}$ for estimating the LLRs of the bits $x_{0}$ of the BL $L_{0}$. Then, the resultant extrinsic LLR information of the BL $L_{0}$ will be input to the "VND1-VND3-CND-VND2" block of Fig. 1 for extracting the a-priori LLRs $L_{a}\left(x_{1}^{i}\right)^{4}$ of the EL $L_{1}$, which is carried out by following the processing of the LLRs in the VND 1, VND 3, CND and VND 2 components of Fig. 2. Specifically, the "VND1-VND3-CND-VND2" block of Fig. 1 performs the following operations step-by-step:

1) VND 1 generates the information of BL $L_{0}$ for VND 3. The extrinsic LLR $L_{e}\left(x_{0}^{i}\right)$ generated by the FEC decoder 0 is input to the VND 1 block of Fig. 1, which extracts the extrinsic LLR information $L_{e}\left(x_{0}^{i}\right)$ and forwards it to the VND 3 block of Fig. 1. Since VND 1 has two input branches, it simply duplicates the soft information $L_{e}\left(x_{0}^{i}\right)$.

2) VND 3 generates the information of $\mathrm{BL} L_{0}$ for CND. Generally, the summation of the soft information $y_{0}$ and the extrinsic LLR $L_{e}\left(x_{0}^{i}\right)$ from VND 1 will be output to $\mathrm{CND}$.

3) CND generates the information of layer $L_{1}$ for VND 2. The LLR information $L_{e}\left(x_{0}^{i}\right)$ and the received check information $y_{01}$ is input to the CND block of Fig. 1 for extracting the LLR information of the systematic bit $x_{1}^{i}$, namely the soft input $L_{a}\left(x_{1}^{i}\right)$ of VND 2 .

4) VND 2 generates the information of EL $L_{1}$ for FEC decoder 1 . The LLR information $L_{a}\left(x_{1}^{i}\right)$ extracted by the CND is input to the VND 2 block of Fig. 1, which extracts the LLR information $L_{a}\left(x_{1}^{i}\right)$ input to the FEC decoder 1 of Fig. 1.

\footnotetext{
${ }^{4}$ The subscripts "a" and "e" in $L_{a}$ and $L_{e}$ stand for the apriori information and extrinsic information [40], respectively.
} 
Then, the FEC decoder 1 of Fig. 1 will decode the EL $L_{1}$ with the aid of the resultant a-priori LLR $L_{a}\left(x_{1}^{i}\right)$ and of the soft parity information received from the channel, namely $y_{1, p}$ of Fig. 1. Similar to the "VND1-VND3-CND-VND2" process, the "VND2-CND-VND3-VND1" process is capable of generating the soft information for the BL. With the aid of the "VND1-VND3-CND-VND2" process and the "VND2CND-VND3-VND1" process, the FEC decoder 0 and FEC decoder 1 may exchange extrinsic information with each other, which constitute an iteration, where the iterative IL decoding process continues until the affordable number of iterations is exhausted or the BL $L_{0}$ is perfectly recovered.

\section{OPTIMIZED FEC CODING RATES}

In this section, we focus our attention on the "Code Rate Optimization" block of our scalable video system seen in Fig. 1, where IL-FEC coded UEP is employed for the sake of achieving an improved video quality. Specifically, the "Code Rate Optimization" block of Fig. 1 characterizes the receiver of Fig. 1 and assists the transmitter in finding the specific FEC coding rates for the layers $L_{0}$ and $L_{1}$ by minimizing the video distortion. Additionally, although having only two layers is assumed in Section II, more complex coding scenarios may be readily considered, as evidenced by the results of Section IV. Moreover, in our system each layer will be mapped to a single packet. Specifically, in the SVC of H.264 coded video scenario, a network abstraction layer unit (NALU) is packed into a single packet, but the different NALUs may carry a different number of bits. Note that in practice several NALUs may be encapsulated into a single packet, depending on the specific requirements of the network.

Below, we will use a classic RSC codec for describing the proposed solution, which aims for minimizing the reconstructed video distortion at the receiver. This is achieved by characterizing the behavior of the receiver seen in Fig. 1. However, the employment of our proposed techniques is not limited to the RSC codec. Firstly, we define the following notations that will aid our analysis:

- $d\left(L_{0}\right)$ : the video distortion induced by the corruption of the BL $L_{0}$, which is measured using the peak signal-tonoise ratio (PSNR);

- $d\left(L_{1}\right)$ : the PSNR distortion, when the BL $L_{0}$ is correct while the EL $L_{1}$ is corrupted;

- $\left|L_{i}\right|$ : the length of the bitstream of layer $L_{i}, 0 \leq i \leq 1$;

- $R$ : the overall coding rate of the system shown in Fig. 1;

- $r_{i}$ : the coding rate of layer $L_{i}, 0 \leq i \leq 1$;

- $p\left(L_{0}\right)$ : the packet error ratio (PER) of layer $L_{0}$;

- $p\left(L_{1}\right)$ : the PER of layer $L_{1}$, when the layer $L_{0}$ is correctly decoded.

According to the IL-FEC decoding process detailed in Section II, both the FEC decoders 0 and 1 of Fig. 1 affect the PER $p\left(L_{0}\right)$, where FEC decoder 1 feeds back a-priori information about BL $L_{0}$ to the FEC decoder 0 through the "VND2-CNDVND3-VND1" blocks of Fig. 1. Specifically, the performance of the FEC decoder 0 depends on $S N R, r_{0},\left|L_{0}\right|$, while the performance of the FEC decoder 1 depends on $S N R, r_{1},\left|L_{1}\right|$. Hence $p\left(L_{0}\right)$ depends on the parameters $S N R,\left|L_{0}\right|,\left|L_{1}\right|, r_{0}$

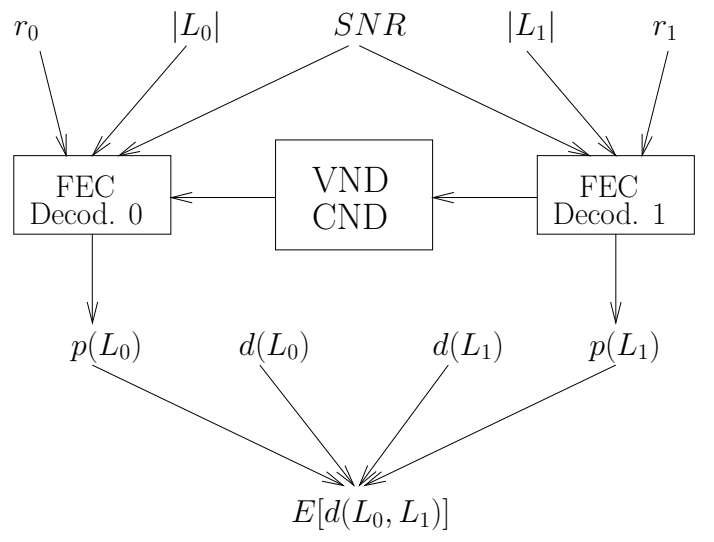

Fig. 3: Dependency chains for calculating the expected distortion formulated in Eq. (4).

and $r_{1}$, which can be expressed as

$$
p\left(L_{0}\right)=f_{0}\left(S N R,\left|L_{0}\right|,\left|L_{1}\right|, r_{0}, r_{1}\right) .
$$

On the other hand, $p\left(L_{1}\right)$ purely depends on the FEC decoder 1 , hence it is determined by the parameters $S N R,\left|L_{1}\right|$ and $r_{1}$, which may be expressed as

$$
p\left(L_{1}\right)=f_{1}\left(S N R,\left|L_{1}\right|, r_{1}\right) .
$$

Given the specific layers $L_{0}$ and $L_{1}, d\left(L_{i}\right)$ may be calculated experimentally as the PSNR degrades due to the erasure of layer $L_{i}$ [20]. Then the expected PSNR distortion induced by BL $L_{0}$ may be estimated as $p\left(L_{0}\right) \cdot d\left(L_{0}\right)$. Additionally, when BL $L_{0}$ is correctly decoded, the expected PSNR distortion induced by EL $L_{1}$ may be estimated as $p\left(L_{1}\right) \cdot d\left(L_{1}\right)$. Hence the expected distortion jointly induced by the decoded layers $L_{0}$ and $L_{1}$, denoted as $E\left[d\left(L_{0}, L_{1}\right)\right]$, may be estimated as

$$
E\left[d\left(L_{0}, L_{1}\right)\right]=p\left(L_{0}\right) \cdot d\left(L_{0}\right)+\left[1-p\left(L_{0}\right)\right] \cdot p\left(L_{1}\right) \cdot d\left(L_{1}\right) .
$$

Based on the above discussions, the expected distortion $E\left[d\left(L_{0}, L_{1}\right)\right]$ may be calculated by following the dependency chains displayed in Fig. 3, which relies on the parameters $S N R,\left|L_{0}\right|, d\left(L_{0}\right), r_{0},\left|L_{1}\right|, d\left(L_{1}\right)$ and $r_{1}$. Among these parameters, $S N R,\left|L_{0}\right|,\left|L_{1}\right|$ are determined by the specific layers $L_{0}, L_{1}$ and the specific transmit power, while $d\left(L_{0}\right)$ and $d\left(L_{1}\right)$ are gleaned from experiments [20]. Hence the coding rates $r_{0}, r_{1}$ determine the distortion $E\left[d\left(L_{0}, L_{1}\right)\right]$ expected for the layers $L_{0}, L_{1}$. Therefore, the objective of the "Code Rate Optimization" block seen in Fig. 1 is to find the specific rates $r_{0}$ and $r_{1}$ capable of minimizing the expected distortion $E\left[d\left(L_{0}, L_{1}\right)\right]$ of Eq. (4), which may be expressed as

$$
\underset{r_{0}, r_{1}}{\arg \min }\left\{E\left[d\left(L_{0}, L_{1}\right)\right]\right\} \text {, }
$$

subject to the condition of

$$
\frac{\left|L_{0}\right|}{r_{0}}+\frac{\left|L_{1}\right|}{r_{1}}=\frac{\left|L_{0}\right|+\left|L_{1}\right|}{R} .
$$

As illustrated in Fig. 3, Eq. (4) relies on the PER definitions of Eqs. (2) and (3), which cannot be theoretically solved. Below we propose our solution of Eqs. (2) and (3) with the assistance of LUTs and the MI, where LUTs are employed 
for characterizing the system components of Fig. 1 and MI is employed for numerically quantifying the reliability of the LLRs. Specifically, Section III-A introduces the preliminaries of solving Eqs. (2) and (3), including the LUTs, the MI flow of the VND and CND, followed by the PER estimation of the BL and EL in Section III-B. Then, we exploit both the estimated PERs $p(\cdot)$ and the video distortions $d(\cdot)$ for determining the optimized coding rates in Section III-C. Finally, Section III-D discusses the overheads imposed by the proposed techniques.

\section{A. Preliminaries}

The receiver of Fig. 1 consists of the following components: demodulator, FEC decoder, VND, CND. The characteristics of these components jointly determine the PER $p\left(L_{0}\right)$ and $p\left(L_{1}\right)$, as shown in Fig. 3. However, neither the demodulator and nor the FEC decoder may be readily characterized theoretically for diverse system configurations, such as different transceivers, FEC generator polynomials, decoding metrics etc. In order to propose a more universal solution, below LUTs are employed for the sake of characterizing both the demodulator and the FEC decoder, while the MI flow of the VND and CND will be detailed afterwards.

1) Lookup Tables: In our analysis we consider a RSCcoded BPSK-modulated system for transmissions over a nondispersive uncorrelated Rayleigh fading wireless channel. Specifically, we model BPSK based transmissions over a Rayleigh fading wireless channel as a function of the channel SNR and generate the demodulator's output LLRs, where LLRs are quantified by the MI value, as in [41]. Furthermore, since the MI invoked for quantifying the reliability of the soft information can be applied for Gaussian distributed LLRs [41] generated by arbitrary transceivers, our procedure may be deemed generically applicable, provided that the transceivers generate near-Gaussian distributed $L L R s^{5}$. By contrast, the RSC codec is modeled as a function of the SNR, of the LLRs of the systematic bits and of the coding rate, while generating the RSC decoder's output extrinsic LLRs and estimating the PER of the RSC decoded packets, where the LLRs are quantified by the MI value. The following LUTs are created for modeling the transceiver and the RSC codec:

- $T_{b}(S N R)$ : The MI value of the LLRs output by the BPSK demodulator for variable channel SNRs, as exemplified in Table IIa. Since this table relies on the $S N R$ only, it may be stored in a one-dimensional space, where the LUTs' size requirements will be detailed in Section III-D.

- $T_{e}\left(S N R, I_{s}, r\right)$ : The MI value of the extrinsic LLR output of the RSC decoder recorded for variable channel SNRs, as exemplified in Table IIb, where $I_{s}$ represents the MI value between the a-priori LLRs of the systematic information and the corresponding information bits, while $r$ represents the coding rate of the RSC codec. Since this table relies on three parameters, it may be visualized

\footnotetext{
${ }^{5}$ Our experiments, not included here for reasons of space-economy, suggest that higher order Quadrature Amplitude Modulation (QAM) can be readily applied for transmission over Rayleigh fading wireless channels, since it generates near-Gaussian distributed LLRs.
}

in a three-dimensional space, where the LUTs' size requirements will also be detailed in Section III-D.

- $T_{p}\left(S N R, I_{s}, r\right)$ : The PER value associated with the LLRs output by the RSC decoder at diverse channel SNRs, as exemplified in Table IIb, where $I_{s}$ represents the MI between the a-priori LLRs of the systematic information and the corresponding information bits, while $r$ represents the coding rate of the RSC codec. Note that this LUT may be generated simultaneously with the LUT $T_{e}\left(S N R, I_{s}, r\right)$, since they have the same input variables.

2) MI Flow of the VND: As seen in Fig. 2a, the operation of the VND may be characterized as $L_{o_{3}}\left(u_{1}\right)=$ $L_{i_{1}}\left(u_{1}\right)+L_{i_{2}}\left(u_{1}\right)$. Assuming that the inputs $L_{i_{1}}\left(u_{1}\right)$ and $L_{i_{2}}\left(u_{1}\right)$ of the VND have the MI values of $I_{i_{1}}\left[u_{1} ; L_{i_{1}}\left(u_{1}\right)\right]$ and $I_{i_{2}}\left[u_{1} ; L_{i_{2}}\left(u_{1}\right)\right]$ respectively, the MI value of the output $L_{o_{3}}\left(u_{1}\right)$ may be expressed as [42]

$$
\begin{aligned}
& I_{o_{3}}\left[u_{1} ; L_{o_{3}}\left(u_{1}\right)\right] \\
& =J\left(\sqrt{J^{-1}\left(I_{i_{1}}\left[u_{1} ; L_{i_{1}}\left(u_{1}\right)\right]\right)^{2}+J^{-1}\left(I_{i_{2}}\left[u_{1} ; L_{i_{2}}\left(u_{1}\right)\right]\right)^{2}}\right),
\end{aligned}
$$

where $J(\cdot)$ and $J^{-1}(\cdot)$ are defined in [37].

3) MI Flow of the CND: Below, we firstly discuss the MI flow of the CND of Fig. 2b, which is designed for the layers $L_{0}$ and $L_{1}$, both carrying the same number of bits. Then we will discuss the practical CND employed for realistic unequal length layers.

As seen in Fig. 2b, the action of the CND can be expressed as $L\left(u_{3}=u_{1} \oplus u_{2}\right)=L\left(u_{1}\right) \boxplus L\left(u_{2}\right)$ [38], where $u_{1}, u_{2}$ and $u_{3}=u_{1} \oplus u_{2}$ are random binary variables. Assuming that the inputs $L_{i}\left(u_{1}\right), L_{i}\left(u_{2}\right)$ of the CND have the MI values of $I_{i}\left[u_{1} ; L\left(u_{1}\right)\right]$ and $I_{i}\left[u_{2} ; L\left(u_{2}\right)\right]$ respectively, the MI value $I_{o}\left[u_{3} ; L\left(u_{3}\right)\right]$ of $u_{3}$ has not been theoretically derived in the literature and since this is not the focus of our work, we model $I_{o}\left[u_{3} ; L\left(u_{3}\right)\right]$ as

$$
I_{o}\left[u_{3} ; L\left(u_{3}\right)\right]=T_{I}\left(I_{i}\left[u_{1} ; L\left(u_{1}\right)\right], I_{i}\left[u_{2} ; L\left(u_{2}\right)\right]\right),
$$

which is also characterized using LUT.

Given the MI $I_{o}\left[u_{3} ; L\left(u_{3}\right)\right]$ in Eq. (8), we now discuss the practical scenario of the CND, where $L_{0}$ and $L_{1}$ may contain an unequal number of bits. As detailed in [31], the encoding architecture of $L_{0}$ and $L_{1}$ may be categorized into two scenarios, namely $\left|L_{0}\right|>\left|L_{1}\right|$ and $\left|L_{1}\right|>\left|L_{0}\right|$. Below we consider the scenario of generating $L\left(x_{1}^{i}\right)$, when $\left|L_{0}\right|>\left|L_{1}\right|$, which can be generalized to other scenarios. As detailed in [31], the bit $x_{01}^{i}$ may be generated as

$$
x_{01}^{i}=x_{1}^{i} \oplus \sum_{j=1}^{n} \oplus x_{0}^{j},
$$

where $\oplus$ denotes the binary XOR operation. Additionally, since on average ${ }^{6}\left|L_{0}\right|$ bits of $L_{0}$ were allocated to the $\left|L_{1}\right|$ bits of $L_{1}$ for generating the bits $x_{01}$, we have $n \in$ $\left\{\left[\frac{\left|L_{0}\right|}{\left|L_{1}\right|}\right],\left[\frac{\left|L_{0}\right|}{\left|L_{1}\right|} \mid-1\right\}\right.$ [31] for Eq. (9). More specifically, $\left(N-\frac{\left|L_{0}\right|}{\left|L_{1}\right|}\right)$ proportion of the bits in the EL $L_{1}$ are encoded based on Eq. (9) using $n=N-1$, while the remaining bits of the EL $L_{1}$ are encoded based on Eq. (9) relying on $n=N$,

\footnotetext{
${ }^{6}$ On average, each bit of $L_{1}$ is encoded by $\frac{\left|L_{0}\right|}{\left|L_{1}\right|}$ bits of $L_{0}$ for generating a single bit of $x_{01}$, as detailed in [31].
} 
where we have $N=\left\lceil\frac{\left|L_{0}\right|}{\left|L_{1}\right|}\right]$.

Based on Eq. (9), the corresponding LLRs $L\left(x_{1}\right)$ of the EL $L_{1}$ may be expressed as

$$
L\left(x_{1}^{i}\right)=L\left(x_{01}^{i}\right) \boxplus \sum_{j=1}^{n} \boxplus L\left(x_{0}^{j}\right) .
$$

Then, the LLRs of $L\left(x_{1}\right)$ are constituted by a mixture of LLRs $L^{N}\left(x_{1}\right)$ and $L^{N-1}\left(x_{1}\right)$, which are calculated based on Eq. (10) in conjunction with $n=N$ and $n=$ $N-1$, respectively. We denote the MI values corresponding to the LLRs $L^{N}\left(x_{1}\right)$ and $L^{N-1}\left(x_{1}\right)$ as $I^{N}\left[x_{1} ; L\left(x_{1}\right)\right]$ and $I^{N-1}\left[x_{1} ; L\left(x_{1}\right)\right]$. Based on Eq. (8), the MI values $I^{N}\left[x_{1} ; L\left(x_{1}\right)\right]$ and $I^{N-1}\left[x_{1} ; L\left(x_{1}\right)\right]$ may be recursively calculated as in Eq. (11), where $1 \leq k \leq N$. Empirically, we assume that the LLRs $L^{N}\left(x_{1}\right)$ and $L^{N-1}\left(x_{1}\right)$ are nearGaussian distributed, while their variance may be denoted as $J^{-1}\left(I^{N}\left[x_{1} ; L\left(x_{1}\right)\right]\right)$ and $J^{-1}\left(I^{N-1}\left[x_{1} ; L\left(x_{1}\right)\right]\right)$, respectively [37]. Again we assume that $L\left(x_{1}\right)$ exhibits nearGaussian distribution, hence the variance of $L\left(x_{1}\right)$ may be formulated as

$$
\begin{aligned}
\sigma^{2} & =D\left(L\left(x_{1}\right)\right) \\
& =\left(1-N+\frac{\left|L_{0}\right|}{\left|L_{1}\right|}\right) \cdot D\left(L^{N}\left(x_{1}\right)\right) \\
& +\left(N-\frac{\left|L_{0}\right|}{\left|L_{1}\right|}\right) \cdot D\left(L^{N-1}\left(x_{1}\right)\right) \\
& =\left(1-N+\frac{\left|L_{0}\right|}{\left|L_{1}\right|}\right) \cdot J^{-1}\left(I^{N}\left[x_{1} ; L\left(x_{1}\right)\right]\right)^{2} \\
& +\left(N-\frac{\left|L_{0}\right|}{\left|L_{1}\right|}\right) \cdot J^{-1}\left(I^{N-1}\left[x_{1} ; L\left(x_{1}\right)\right]\right)^{2} .
\end{aligned}
$$

Finally, the MI value of $L\left(x_{1}\right)$ may be expressed using the $J$ function as in Eq. (13).

\section{B. PER Estimation}

The PER of the decoded BL and EL having coding rates of $r_{0}$ and $r_{1}$ will be estimated in Section III-B.1 and Section III-B.2, respectively.

1) IL-FEC Coded BL: As shown in Fig. 3, the PER of the BL $L_{0}$ depends on the characteristics of the IL-FEC decoder, including the generator polynomials, the trellis decoding techniques employed, as well as the VND and the CND of Fig. 2. Below we propose our solution conceived for estimating the PER of Eq. (3) with the aid of the LUTs defined in Section III-A.1.

The PER estimation flow-chart of the IL-FEC coded BL is illustrated in Fig. 4, which follows the decoding process detailed in Section II. Given the $S N R$, the MI value $I_{s}$ of $y_{0}$ can be generated by the LUT $T_{b}(S N R)$. Furthermore, the MI value of the extrinsic LLR gleaned from the RSC decoder 0 may be expressed as $T_{e}\left[S N R, T_{b}(S N R), r_{0}\right]$. Then the "VND1-VND3-CND-VND2" process of Fig. 4 is capable of calculating the MI value $I_{a}\left(x_{1}\right)$ of $L_{a}\left(x_{1}^{i}\right)$. Afterwards, based on the LUT $T_{e}\left(S N R, I_{s}, r\right)$ of the RSC decoder 1, the MI value $I_{e}\left(x_{1}\right)$ of the extrinsic information may be readily expressed as

$$
I_{e}\left(x_{1}\right)=T_{e}\left[S N R, T_{b}(S N R), r_{1}\right] .
$$

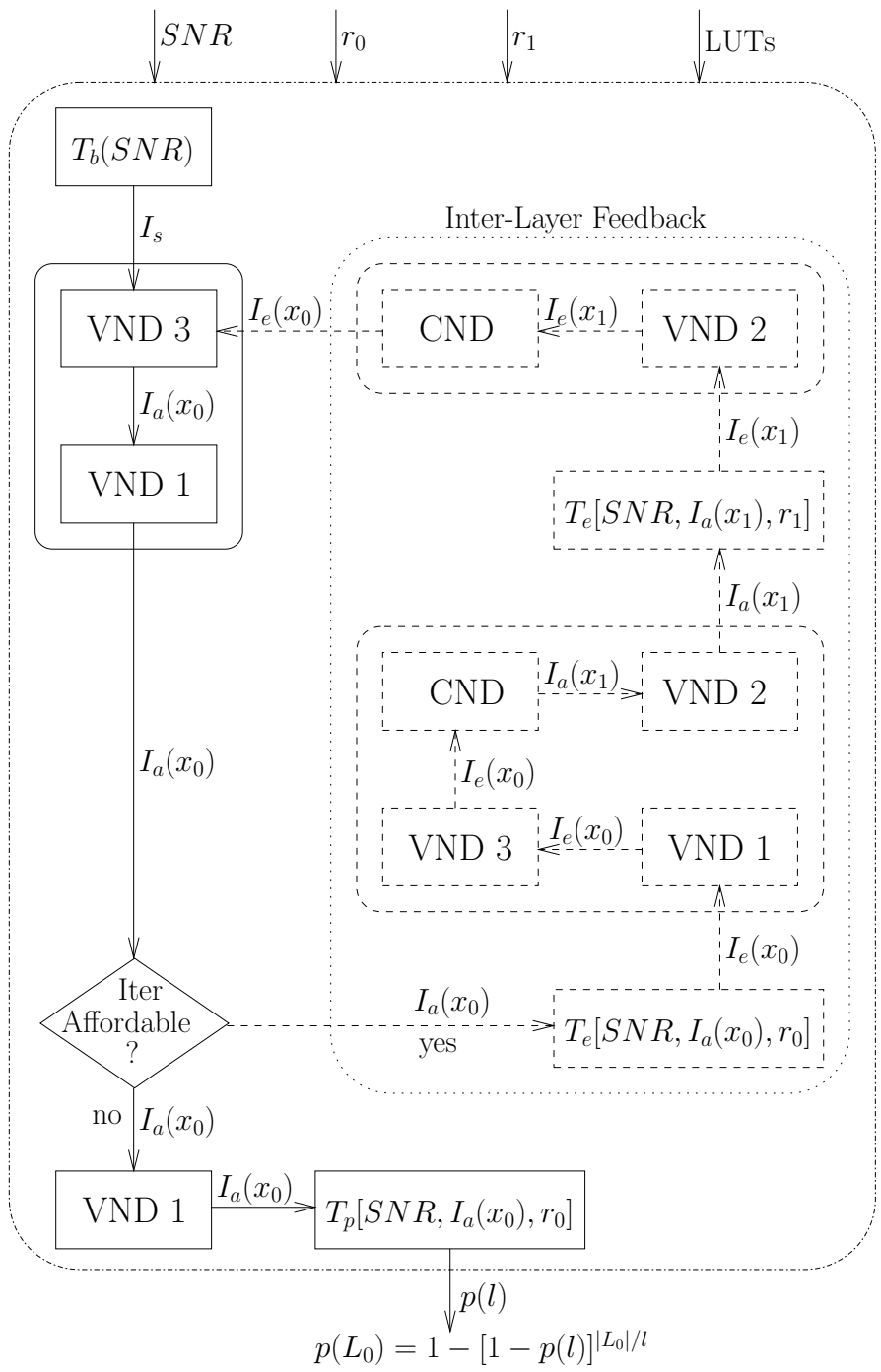

Fig. 4: Flow-chart of the PER estimation for the IL-FEC coded BL $L_{0}$, where $p\left(L_{0}\right)$ will be employed by the "Code Rate Optimization" block of Fig. 1 for resolving the objective function of Eq. (5).

Then, following the "VND2-CND-VND3-VND1" process of Fig. 4, the improved MI value $I_{a}\left(x_{0}\right)$ of $L_{a}\left(x_{0}^{i}\right)$ can be calculated. Finally, the PER associated with the packet length of $l^{7}$, denoted as $p(l)$, may be estimated as $T_{p}\left[S N R, I_{a}\left(x_{0}\right), r_{0}\right]$, which is also shown in Fig. 4. Below, we now detail the method of deriving the PER $p\left(L_{0}\right)$ of the BL $L_{0}$ from $p(l)=$ $T_{p}\left[S N R, I_{a}\left(x_{0}\right), r_{0}\right]$.

The burst error distribution of RSC codec has been investigated in [43], which is independent of the packet length. Let us consider a RSC-decoded packet having a length of $\left(n_{1} \times n_{2}\right)$ bits. Then this packet may be partitioned in two ways, as displayed in Fig. 5. Specifically, it may be divided into $n_{1}$ packets, each carrying $n_{2}$ bits or $n_{2}$ packets associated with $n_{1}$ bits each. Assuming that $p\left(n_{i}\right)$ indicates the PER of the $n_{i}$-bit packet, based on Fig. 5a the PER $p\left(n_{1} \cdot n_{2}\right)$ may

\footnotetext{
${ }^{7}$ The packet containing $l$ bits is employed for generating the LUT $T_{p}\left[S N R, I_{a}\left(x_{0}\right), r_{0}\right]$.
} 


$$
I^{k}\left[x_{1} ; L\left(x_{1}\right)\right]= \begin{cases}T_{I}\left(I\left[x_{0} ; L\left(x_{0}\right)\right], I\left[x_{01} ; L\left(x_{01}\right)\right]\right), & k=1, \\ T_{I}\left(I^{k-1}\left[x_{1} ; L\left(x_{1}\right)\right], T_{I}\left(I\left[x_{0} ; L\left(x_{0}\right)\right], I\left[x_{01} ; L\left(x_{01}\right)\right]\right)\right), & k>1 .\end{cases}
$$

$$
I\left[x_{1} ; L\left(x_{1}\right)\right]=J(\sigma)=J\left(\sqrt{\left(1-N+\frac{\left|L_{0}\right|}{\left|L_{1}\right|}\right) \cdot J^{-1}\left(I^{N}\left[x_{1} ; L\left(x_{1}\right)\right]\right)^{2}+\left(N-\frac{\left|L_{0}\right|}{\left|L_{1}\right|}\right) \cdot J^{-1}\left(I^{N-1}\left[x_{1} ; L\left(x_{1}\right)\right]\right)^{2}}\right) .
$$

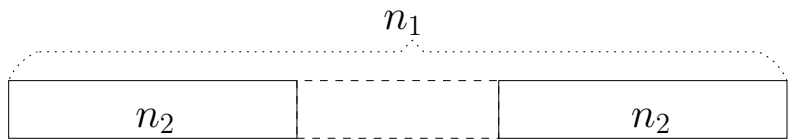

(a) $n_{1} \times n_{2}$

$n_{2}$

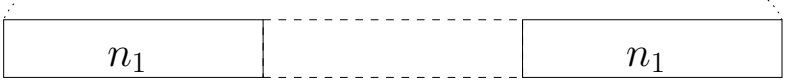

(b) $n_{2} \times n_{1}$

Fig. 5: A packet having a length of $\left(n_{1} \times n_{2}\right)$ bits may be divided into $n_{1} / n_{2}$ shorter packets with a length of $n_{2} / n_{1}$ bits.

be estimated as

$$
p\left(n_{1} \cdot n_{2}\right)=1-\left[1-p\left(n_{2}\right)\right]^{n_{1}},
$$

where $p\left(n_{2}\right)$ is the PER of the $n_{1}$ packets of Fig. 5a. Similarly, we have $p\left(n_{1} \cdot n_{2}\right)=1-\left[1-p\left(n_{1}\right)\right]^{n_{2}}$ based on Fig. 5b. Then, for arbitrary numerical values of $n_{1}, n_{2}$ we have

$$
p\left(n_{1}\right)=1-\left[1-p\left(n_{2}\right)\right]^{n_{1} / n_{2}} .
$$

Upon assuming that $n_{1}, n_{2}$ of Eq. (16) are given by $\left|L_{0}\right|$ and $l$, respectively, the PER $p\left(L_{0}\right)$ of the BL $L_{0}$ may be estimated as

$$
\begin{aligned}
p\left(L_{0}\right) & =f_{0}\left(S N R,\left|L_{0}\right|,\left|L_{1}\right|, r_{0}, r_{1}\right) \\
& =1-\left[1-T_{p}\left[S N R, I_{a}\left(x_{0}\right), r_{0}\right]\right]^{\left|L_{0}\right| / l},
\end{aligned}
$$

where $l$ is the packet length employed for generating the LUT $T_{p}$.

For visualizing the accuracy of our proposed PER estimation for the BL, we assume $\left|L_{0}\right|=2000, r_{0}=0.5$ and $R=$ 0.5 , while the RSC codec is configured by the generator polynomials of $[1011,1101,1111]$. Diverse EL packet length of $L_{1}$ are tested. The resultant simulated and estimated PERs of the BL $L_{0}$ are displayed in Fig. 6 for different $S N R$ values. As observed in Fig. 6, the estimated PER does not perfectly match the simulated PER, but it closely follows the same trend, especially in the PER region above $10^{-2}$, which is our area of interest. Note that the estimation error may be introduced both by the LUTs and by the near-Gaussian distributed LLRs output by the demodulator.

2) FEC Coded EL: When the BL $L_{0}$ is correctly decoded, infinite LLRs will be input to the CND 3 of Fig. 1 [31], hence the LLRs having the MI value of $I_{s}$ will be input to the VND

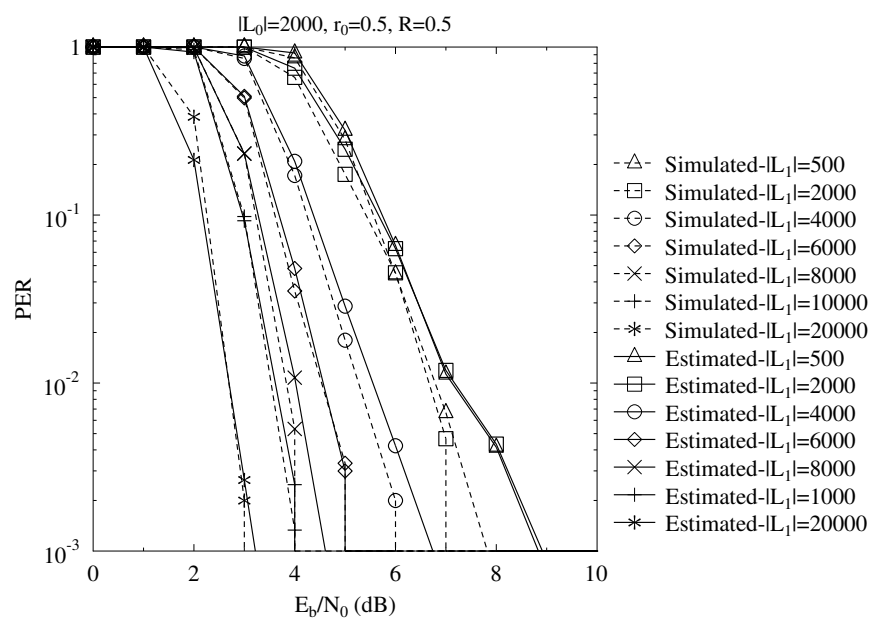

Fig. 6: Comparison of the simulated and estimated PER of the $\mathrm{BL}$, where the parameters $\left|L_{0}\right|=2000, r_{0}=0.5, R=0.5$ are employed.

2 by the CND. Therefore the PER of the EL having a length of $C$ bits may be estimated as $T_{p}\left(S N R, I_{s}, r_{1}\right)$ with the aid of the LUT $T_{p}$. Then, similar to the Eq. (17), the PER $p\left(L_{1}\right)$ of the EL $L_{1}$ may be finally estimated as

$$
\begin{aligned}
p\left(L_{1}\right) & =f_{1}\left(S N R,\left|L_{1}\right|, r_{1}\right) \\
& =1-\left[1-T_{p}\left(S N R, I_{s}, r_{1}\right)\right]^{\left|L_{1}\right| / l},
\end{aligned}
$$

where $l$ is the packet length employed for generating the LUT $T_{p}$.

For visualizing the accuracy of our proposed EL PER estimation, we assume $r_{1}=0.33$, while the RSC codec is configured by the generator polynomials of $[1011,1101,1111]$. Moreover, we consider $\left|L_{1}\right|$ values ranging from 80 to 4000 , while the LUT $T_{p}\left(S N R, I_{s}, r_{1}\right)$ is generated based on $l=$ 1000. The resultant simulated and estimated PER of the EL $L_{1}$ is displayed in Fig. 7. Observe in Fig. 7 that the estimation error is much smaller than that in Fig. 6 due to the fact that the EL is protected by an RSC codec without using IL techniques.

\section{Optimized Coding Rates}

Based on Section III-B, the PERs of $L_{0}$ and $L_{1}$ are estimated according to the specified coding rates of $r_{0}$ and $r_{1}$ for the BL and EL, respectively. Hence the expected distortion $E\left[d\left(L_{0}, L_{1}\right)\right]$ imposed by the wireless transmission using rates of $r_{0}$ and $r_{1}$ may be readily calculated using Eq. (4). In order to find the rates $r_{0}, r_{1}$ minimizing the distortion $E\left[d\left(L_{0}, L_{1}\right)\right]$ in Eq. (5), a full search of the coding rate space may be 


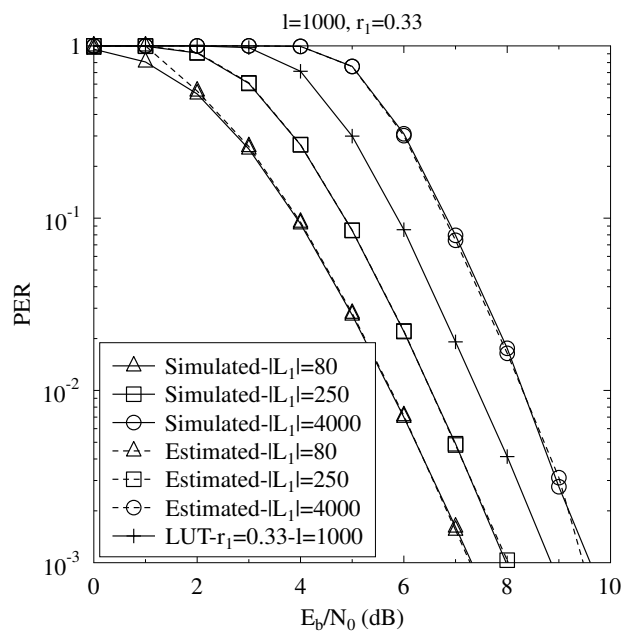

Fig. 7: Comparison of the simulated and estimated PER for the EL, where the parameters $l=1000, r_{1}=0.33$ are employed.

performed. Note that $r_{1}$ is determined by Eq. (6) for a given $r_{0}$, hence the full search is performed in a one-dimensional space to find the optimal $r_{0}$.

To elaborate a little further, we consider the scenario of $S N R=10 d B, R=1 / 3,\left|L_{0}\right|=\left|L_{1}\right|=100, d\left(L_{0}\right)=20$, $d\left(L_{1}\right)=10$. Based on the conditions formulated in Eq. (6), the coding rate $r_{1}$ of $L_{1}$ is determined as follows:

$$
\begin{aligned}
& r_{1}=\frac{\left|L_{1}\right|}{\frac{\left|L_{0}\right|+\left|L_{1}\right|}{R}-\frac{\left|L_{0}\right|}{r_{0}}}=\frac{r_{0}}{6 \cdot r_{0}-1} \\
& r_{0} \leq 1, r_{1} \leq 1 .
\end{aligned}
$$

Then, based on Eqs. (2), (3) and (4) the expected value of $E\left[d\left(L_{0}, L_{1}\right)\right]$ in Eq. (5) may be formulated as

$$
\begin{aligned}
& E\left[d\left(L_{0}, L_{1}\right)\right] \\
& =p\left(L_{0}\right) \cdot d\left(L_{0}\right)+\left[1-p\left(L_{0}\right)\right] \cdot p\left(L_{1}\right) \cdot d\left(L_{1}\right) \\
& =20 \cdot f_{0}\left(10,100,100, r_{0}, \frac{r_{0}}{6 \cdot r_{0}-1}\right) \\
& +10 \cdot\left[1-f_{0}\left(10,100,100, r_{0}, \frac{r_{0}}{6 \cdot r_{0}-1}\right)\right] \\
& \cdot f_{1}\left(10,100, \frac{r_{0}}{6 \cdot r_{0}-1}\right) \\
& =g\left(r_{0}\right),
\end{aligned}
$$

where the functions $f_{0}$ and $f_{1}$ are discussed in the 'PER Estimation' Section III-B. Note that we introduced the shorthand of $E\left[d\left(L_{0}, L_{1}\right)\right]=g\left(r_{0}\right)$ in Eq. (20) for simplicity. Based on Eq. (19), we can readily arrive at $\frac{1}{5} \leq r_{0} \leq 1$. Then we artificially limit $r_{0}$ to the following discrete practical values $[0.2: 0.05: 1]^{8}$. Finally, the solution $r_{0}$ of the objective function $\arg \min \left\{E\left[d\left(L_{0}, L_{1}\right)\right]\right\}$ in Eq. (5) may be readily found to be:

$$
\left\{r_{0} \mid \underset{r_{0}}{\arg \min } g\left(r_{0}\right), r_{0} \in\{0.2: 0.05: 1\}\right\},
$$

which can be solved by performing a one-dimensional full

\footnotetext{
${ }^{8}$ The value of $r_{0}$ ranges from 0.2 to 1 with a constant step of 0.05 .
}

search. Then, given $r_{0}$ the coding rate $r_{1}$ may be readily expressed from Eq. (19) .

\section{Overheads}

All the optimization operations detailed in Section III are carried out at the transmitter. Below, we discuss the overheads imposed at the transmitter, while the overheads imposed at the receiver are given in [31]. The overheads imposed at the transmitter include the estimation of $d(\cdot)$, the generation of LUTs, the estimation of $p(\cdot)$, the imposed frame delay and the full search detailed in Section III-C. Among these overheads, the generation of LUTs only imposes extra offline design-time, while the estimation of $d(\cdot), p(\cdot)$ and the full search impose extra on-line run-time complexity. For notational simplicity, we use $n_{s n r}, n_{I}, n_{r}$ to denote the number of the parameters $S N R, I_{s}, r$, respectively, which are used for generating the LUTs. For example, if the values of $I_{s}$ range from 0 to 1 with a step size of 0.01 , we have $n_{I}=101$. In the following, we will analyze these overheads in order to validate our system.

1) Estimation of $d(\cdot): d\left(L_{i}\right)$ is estimated in a similar manner to the procedure of [20], where the distortion $d\left(L_{0}\right)$ may be obtained by decoding the bitstream in the presence of a corrupted BL $L_{0}$. Alternatively, the solutions of [21], [25], [44] may be applied in our system.

2) Generation of LUTs: Three LUTs were generated in our proposed solution. The LUT $T_{b}(S N R)$ characterizes the channel and transceiver, hence this table has to be regenerated when the channel or the transceiver are changed. However, this LUT only has a single variable, hence it is straightfarward to generate the LUT $T_{b}(S N R)$. Additionally, the LUTs $T_{e}\left(S N R, I_{s}, r\right)$ and $T_{p}\left(S N R, I_{s}, r\right)$ can be simultaneously generated by simulations, since they have the same variables. However, they are independent of the channel and transceiver, but they are dependent on the configuration of the FEC code. Hence the LUTs $T_{e}$ and $T_{p}$ have to be regenerated, when the FEC is reconfigured. Note that all the LUTs are independent of the video sequences and that these LUTs are generated during the design process. The size of these LUTs depends on the variables, hence the LUTs $T_{b}, T_{e}, T_{p}$ have sizes of $n_{s n r},\left(n_{s n r} \cdot n_{I} \cdot n_{r}\right),\left(n_{s n r} \cdot n_{I} \cdot n_{r}\right)$, respectively.

3) Estimation of $p(\cdot)$ : The CND and VND are involved in the PER estimation process, as well as in the search through the LUTs. As detailed in Section III-A, the MI flows of the VND and CND impose a low computational complexity. Furthermore, in our system the values of each variable are chosen using a constant step-size, which guarantees low complexity, while searching through the LUTs. For example, let us assume that the vector $[0: 0.01: 1]$ is employed for representing the values of $I_{s}$ for generating the LUTs. In this case, the $I_{s}$ value of 0.5 can be directly located at index 50 .

4) Full Search of $r_{0}: n_{r}$ coding rates of $r_{0}$ may be tentatively tested, while $r_{1}$ is determined by Eq. (6) for a given $r_{0}$. Hence the complexity of the full search may be expressed as $O\left(C \times n_{r}\right)$, where $C$ is a constant, representing the complexity of estimating $p\left(L_{i}\right)$. However, when more layers are encapsulated in the source bitstream, the full-search 


\begin{tabular}{|l|r|r|}
\hline & Football & Foreman \\
\hline \hline Representation & YUV 4:2:0 & YUV 4:2:0 \\
\hline Format & QCIF & QCIF \\
\hline Bits Per Pixel & 8 & 8 \\
\hline FPS & 15 & 30 \\
\hline Number of Frames & 30 & 30 \\
\hline Video Codec & SVC-H.264 & SVC-H.264 \\
\hline Bitrate & $2297 \mathrm{kbps}$ & $218 \mathrm{kbps}$ \\
\hline Error-Free PSNR & $38.8 \mathrm{~dB}$ & $37.3 \mathrm{~dB}$ \\
\hline Error Concealment & Frame-Copy & Frame-Copy \\
\hline
\end{tabular}

TABLE I: The parameters of the video sequences employed.

based complexity increases exponentially, leading to a multidimensional optimization problem, which has been widely studied in the literature [45]-[47]. Specifically, the adaptive particle swarm optimization (APSO) technique of [47] may be readily employed for finding the global optimum in realtime. Note that in the scenarios where as few as 2-4 layers in the range of 2 to 4 are generated, even elite-force full-search is realistic at a modest complexity.

5) Delay: Our technique is implemented using the SVC of H.264 as detailed in Section IV, where each video frame can be encoded into a single BL and multiple ELs. Since the IL encoding and decoding process is performed within each frame, no extra delay is imposed by our proposed technique. Hence, the system can be applied in low-delay applications.

\section{System Performance}

In this section, we benchmark our proposed Optimized-ILRSC system against an identical RSC-aided system employing the traditional UEP technique. The generator polynomials of $[1011,1101,1111]$ are employed for configuring the RSC codec. Moreover, BPSK modulated signals were transmitted through non-dispersive uncorrelated Rayleigh fading wireless channels. The 30-frame Foreman and Football video clips represented in $(176 \times 144)$-pixel quarter common intermediate format (QCIF) and 4:2:0 YUV format were encoded by the JSVM H.264/AVC reference video codec. The Football and Foreman sequences exhibit different motion-activity, hence they allow us to demonstrate the universal nature of our proposed system. These sequences were scanned at 15 and 30 FPS, respectively. The "frame-copy" based error concealment tool built into the JSVM H.264/AVC reference codec was activated for combating the effects of channel impairments. The GOP duration was set to 15, hence an Intra-frame-coded (I) picture was inserted every 15 frames. Correspondingly, both of the two video sequences were encoded into GOPs, consisting of an I frame, followed by 14 predicted (P) frames.
Since the bi-directionally predicted (B) frames may impose error propagation on their forward- and backward-predicted dependent-frames, the B frames are disabled in our JSVM configuration. Additionally, only the Medium Grain Scalability (MGS) [4], [48] feature is enabled, when encoding the video sequences into three different-quality ELs, namely into the layers $L_{0}, L_{1}$ and $L_{2}$ using the standardized quantization parameters $(\mathrm{QP})$ of 40, 32 and 24, respectively. Furthermore, each frame will be encoded into a single BL and two ELs, resulting in three network abstraction layer units (NALUs). These configurations jointly result in a bitrate of $2297 \mathrm{kbps}$ and a PSNR of $38.8 \mathrm{~dB}$ for the Football sequence in the absence of transmission errors, while achieving 37.3 dB PSNR at 218 kbps for the Foreman sequence. The system parameters are listed in Table I.

Moreover, each SVC H.264/AVC-compressed bitstream was channel encoded and transmitted on a NALU by NALU [5] basis, which is the smallest unit to be decoded by the SVC decoder. Each NALU was protected by CRC codes. At the receiver, each decoded NALU failing to pass the CRC check process was removed before the SVC video decoding process. In all of our experiments, the compressed bitstreams were transmitted 300 times in order to generate statistically sound performance curves.

\section{A. Off-line LUTs Generation}

In our experiments, the vectors of $[0: 0.5: 15]$, $[0: 0.01: 1],[0.33: 0.02: 1]^{9}$ are utilized for the variables $S N R, I_{s}, r$, respectively, for generating the LUTs, which result in $n_{s n r}=31, n_{I}=101, n_{r}=33$. Firstly, we recorded the MI values of the LLRs output by the BPSK demodulator for the SNR values of $[0: 0.5: 15]$ for the sake of generating the LUT $T_{b}(S N R)$. Then for each $S N R$, the MI values $[0: 0.01: 1]$ for $I_{s}$ and the coding rates $[0.33: 0.02: 1]$ for $r$ were utilized for generating the LUTs $T_{e}\left(S N R, I_{s}, r\right)$ and $T_{p}\left(S N R, I_{s}, r\right)$, simultaneously. Furthermore, 8-byte floating values were utilized for storing the LUTs in memory. Correspondingly, the LUTs $T_{b}, T_{e}$ and $T_{p}$ require memory sizes of about 248 bytes, $800 \mathrm{~KB}$ and $800 \mathrm{~KB}$, respectively. Finally, $n_{r}=33$ results in a full search complexity of $\left(C \times n_{r}^{2}=C \cdot 1089\right)$, thereby finding the optimized coding rates of $r_{0}, r_{1}$ and $r_{2}$. A number of entries of the LUTs generated for our system are displayed in Table II.

\section{B. System Performance}

In this section, we benchmark our system against the traditional equal error protection (EEP) system, which is referred to as EEP-RSC. Furthermore, the traditional optimal UEPRSC (Opt-UEP-RSC) system is also presented, which is the system of [20] applied in the scenario of an RSC coded system, where all the layers are unequally protected by an RSC code dispensing with the IL technique. Moreover, the IL aided EEP system (EEP-IL-RSC) is also considered.

The BER curves of the $L_{0}, L_{1}$ and $L_{2}$ layers of the Football sequence are displayed in Figs. 8a, 8b and 8c, respectively,

\footnotetext{
${ }^{9}$ These values can be stored as floats in 8 bytes each.
} 


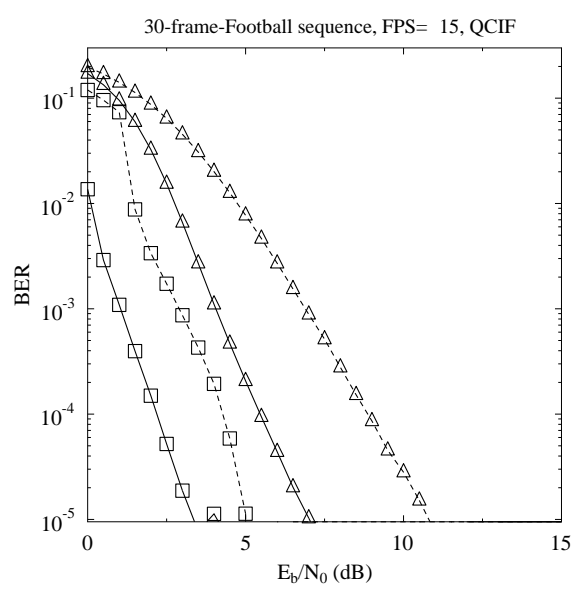

(a) BER vs $E_{b} / N_{0}$ for $L_{0}$-Football

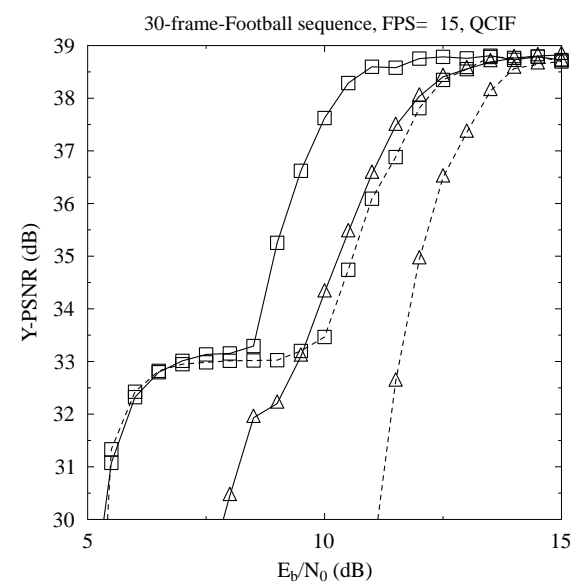

(d) PSNR vs $E_{b} / N_{0}$ for Football

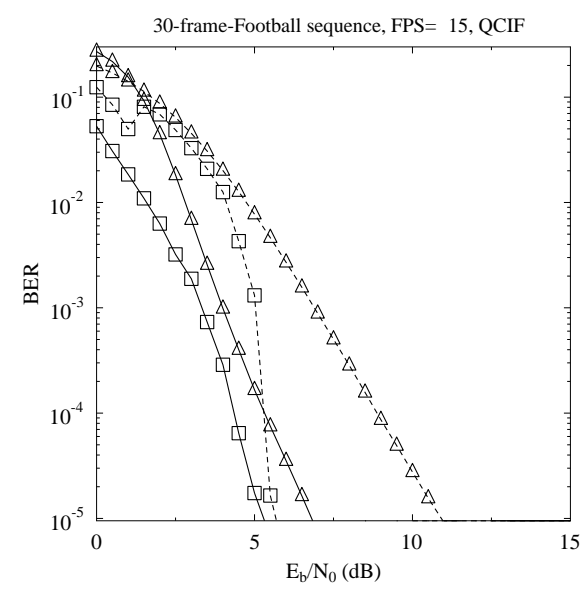

(b) BER vs $E_{b} / N_{0}$ for $L_{1}$-Football

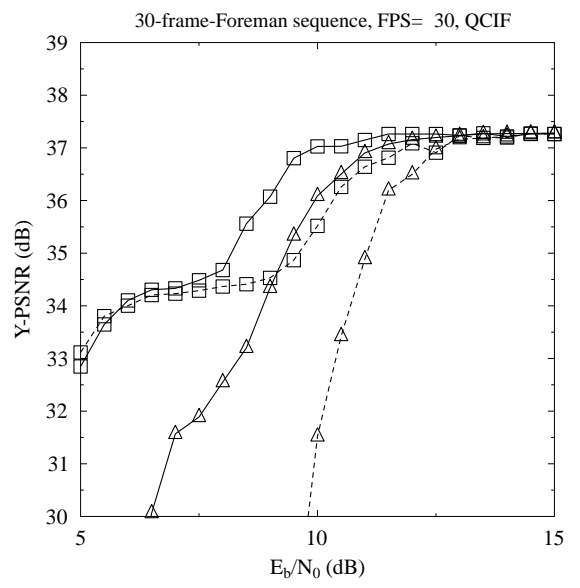

(e) PSNR vs $E_{b} / N_{0}$ for Foreman

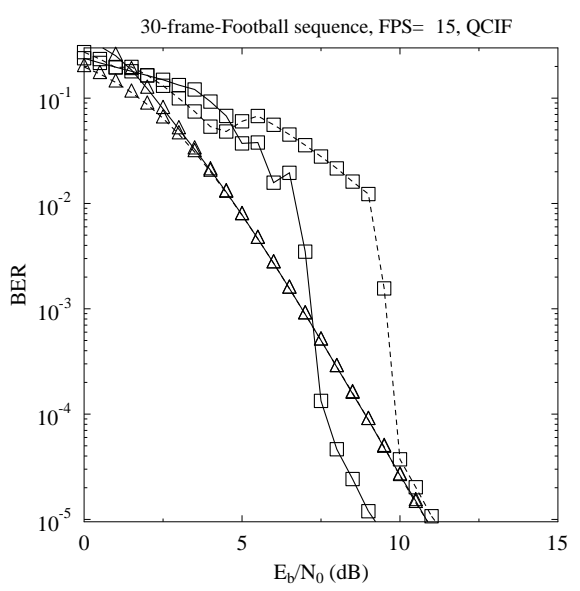

(c) BER vs $E_{b} / N_{0}$ for $L_{2}$-Football

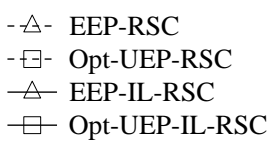

(f) Tested schemes

Fig. 8: BER and PSNR versus $E_{b} / N_{0}$ performance comparison of the proposed system and the benchmarkers, namely the EEP-RSC scheme, the Opt-UEP-RSC scheme [20], the EEP-IL-RSC scheme and the Opt-UEP-IL-RSC scheme for the Football and Foreman sequences. Non-dispersive uncorrelated Rayleigh fading wireless channels

where the Opt-UEP-IL-RSC system always outperforms the Opt-UEP-RSC system. Moreover, as seen in Fig. 8c, the EEP schemes outperform the Opt-UEP-IL-RSC and Opt-UEP-RSC schemes in the lower $E_{b} / N_{0}$ range. This is due to the fact that the coding-rate of layer $L_{2}$ is sacrificed for the sake of protecting the $L_{0}$ and $L_{1}$ layers. Similar trends were observed for the Foreman sequence, which are not included here owing to space-economy.

The PSNR versus $E_{b} / N_{0}$ performance recorded for the Football sequence is displayed in Fig. 8d, where we observe that the EEP-IL-RSC scheme substantially outperforms the EEP-RSC system, while it only slightly outperforms the Opt-UEP-RSC scheme. However, by optimizing the coding rates, the Opt-UEP-IL-RSC scheme becomes capable of substantially outperforming the Opt-UEP-RSC system. In the low $E_{b} / N_{0}$ range, the Opt-UEP-IL-RSC and Opt-UEP-RSC schemes have a similar PSNR performance and tend to exhibit a residual error floor. This is due to the fact that these two systems endeavor to protect the BL $L_{0}$ by sacrificing the protection of the ELs, where the ELs are unlikely to be recovered before reaching $E_{b} / N_{0}=10 \mathrm{~dB}$. This error floor becomes explicit in the BER curves of $L_{2}$ displayed in Fig. 8c. On the other hand, the Opt-UEP-RSC scheme outperforms the EEP-RSC scheme by about $E_{b} / N_{0}=1.3 \mathrm{~dB}$ at a PSNR of $38 \mathrm{~dB}$. The Opt-UEP-IL-RSC scheme achieves an $E_{b} / N_{0}$ reduction of about $1.9 \mathrm{~dB}$ compared to the Opt-UEP-RSC scheme at a PSNR of $38 \mathrm{~dB}$. Alternatively, about $3.3 \mathrm{~dB}$ of PSNR video quality improvement is observed at an $E_{b} / N_{0}$ of $10 \mathrm{~dB}$. A subjective comparison of the benchmarkers recorded for the Football sequence is presented in Fig. 9.

For providing further insights for video scenes having different motion-activity, the PSNR versus $E_{b} / N_{0}$ performance of the Opt-UEP-IL-RSC is portrayed in Fig. 8e using the Foreman sequence. The Opt-UEP-RSC scheme outperforms the EEP-RSC system by about $E_{b} / N_{0}=0.8 \mathrm{~dB}$ at a PSNR of $37 \mathrm{~dB}$. Moreover, about $1.7 \mathrm{~dB}$ of power reduction is achieved by the Opt-UEP-IL-RSC scheme compared to the Opt-UEPRSC scheme at a PSNR of $37 \mathrm{~dB}$. Alternatively, about $1.6 \mathrm{~dB}$ of PSNR video quality improvement may be observed at an $E_{b} / N_{0}$ of $10 \mathrm{~dB}$. 


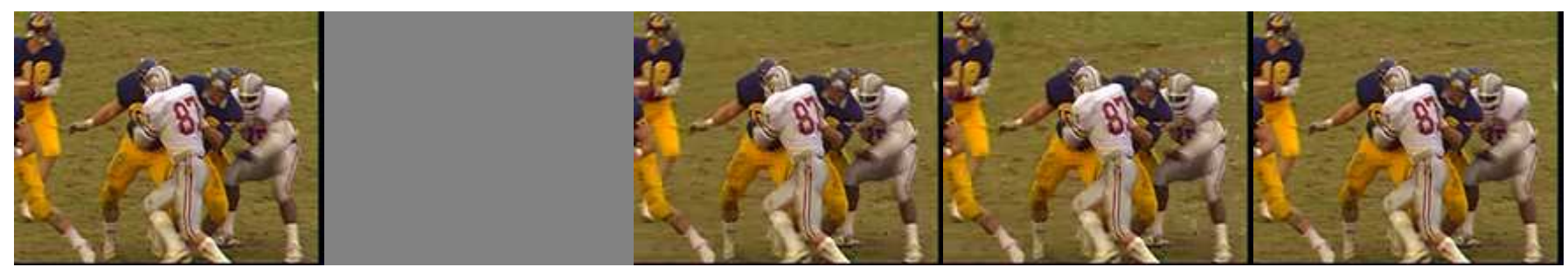

Fig. 9: Comparison of frames at $E_{b} / N_{0}$ of $10 \mathrm{~dB}$ for the Football sequences. The five columns (from left to right) indicate frames of the original video, the EEP-RSC scheme, the Opt-UEP-RSC [20] scheme, the EEP-IL-RSC scheme and the OptUEP-IL-RSC scheme, respectively.

\begin{tabular}{|l|r|}
\hline$S N R$ & $I_{s}$ \\
\hline \hline$\vdots$ & $\vdots$ \\
\hline 1 & 0.45 \\
\hline 2 & 0.50 \\
\hline 3 & 0.56 \\
\hline 4 & 0.62 \\
\hline 5 & 0.67 \\
\hline 6 & 0.72 \\
\hline 7 & 0.76 \\
\hline 8 & 0.80 \\
\hline$\vdots$ & $\vdots$ \\
\hline
\end{tabular}

(a) $T_{b}(S N R)$

\begin{tabular}{|l|l|l|r|r|}
\hline$S N R$ & $I_{a}$ & $r$ & $I_{e}$ & $p(l)$ \\
\hline \hline$\vdots$ & $\vdots$ & $\vdots$ & $\vdots$ & $\vdots$ \\
\hline 3 & 0.67 & 0.87 & 0.81 & 0.93 \\
\hline 3 & 0.67 & 0.88 & 0.83 & 0.89 \\
\hline 3 & 0.67 & 0.89 & 0.85 & 0.84 \\
\hline 3 & 0.67 & 0.90 & 0.87 & 0.77 \\
\hline 3 & 0.67 & 0.91 & 0.89 & 0.69 \\
\hline 3 & 0.67 & 0.92 & 0.91 & 0.57 \\
\hline 3 & 0.67 & 0.93 & 0.93 & 0.46 \\
\hline 3 & 0.67 & 0.94 & 0.94 & 0.33 \\
\hline$\vdots$ & $\vdots$ & $\vdots$ & $\vdots$ & $\vdots$ \\
\hline
\end{tabular}

(b) $T_{e}\left(S N R, I_{s}, r\right)$ and $T_{p}\left(S N R, I_{s}, r\right)$
TABLE II: Example of the LUTs.

\section{Optimized Coding Rates}

The optimized coding rates found by our proposed regime for the Football sequence are shown in Fig. 10 in comparison to the benchmarkers. Specifically, the y axis of Fig. 10 indicates the averaged coding rates of all frames. Furthermore, the coding rates of $L_{0}$ and $L_{2}$ are presented, while the curves of $L_{1}$ are similar to those of the BL $L_{0}$. Observe in Fig. 10 that in the low $E_{b} / N_{0}$ range the coding rates of the BL $L_{0}$ of the Opt-UEP-RSC and Opt-UEP-IL-RSC schemes are lower than the overall coding rate of 0.5 . The reason of this observation is that the protection of the layers $L_{1}$ and $L_{2}$ is sacrificed for the sake of protecting the more important BL $L_{0}$. At high $E_{b} / N_{0}$ values, the coding rates of the $\mathrm{BL} L_{0}$ are increased due to the fact that $L_{0}$ is more likely to be corrected at high coding rates, when favorable channel conditions prevail. In comparison to the BL $L_{0}$, the coding rates of $L_{2}$ are reduced upon increasing $E_{b} / N_{0}$.

\section{CONCLUSions}

We proposed an optimization technique for bit-level ILFEC coded scalable video transmission over wireless channels, where soft-decoding aided FEC is utilized. Firstly, LUTs were generated for the sake of optimizing the wireless transceivers and the FEC codec, which relied on exploiting the off-line training based MI. Moreover, the MI exchange of the VND and CND of Fig. 2 was exploited for realistic video systems, where both the PERs of the IL-FEC coded BL and of the

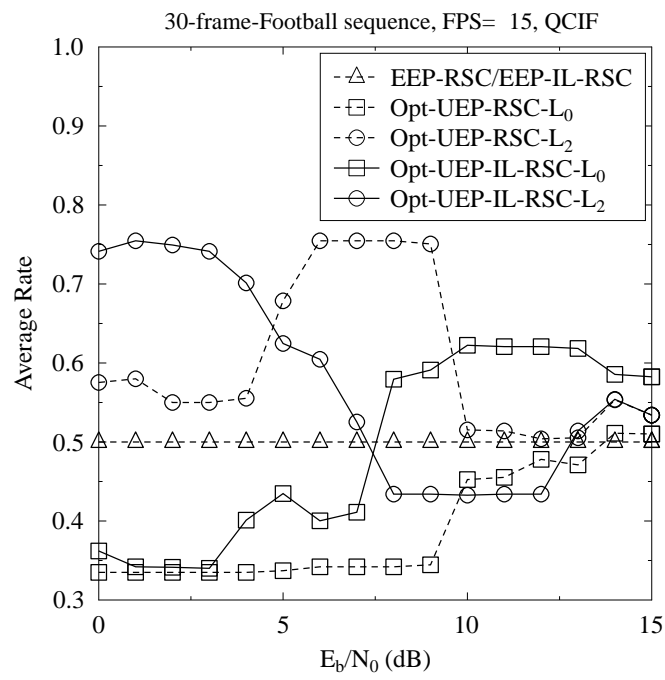

Fig. 10: Coding rates comparison of the benchmarkers, namely the EEP-RSC scheme, the Opt-UEP-RSC [20] scheme, the EEP-IL-RSC scheme and the Opt-UEP-IL-RSC scheme, for the Football sequence. Non-dispersive uncorrelated Rayleigh fading wireless channels

FEC coded ELs were estimated, based on the knowledgeable specific coding rates of all the scalable video layers. Then the expected PSNR distortion was minimized by performing a full search. BPSK modulation, an RSC FEC code and the SVCH.264 video codec were employed in our design example, but our solution may be readily applied for arbitrary transceivers generating near-Gaussian distributed LLRs, for arbitrary noniteratively decoded channel codes and for arbitrary layered video transmissions. Our simulation results show that the proposed optimized IL-FEC system outperforms the traditional optimal UEP system by about $1.9 \mathrm{~dB}$ of $E_{b} / N_{0}$ or $3.3 \mathrm{~dB}$ of PSNR, when employing a RSC code.

In our future work, we will incorporate the IL-FEC scheme into multiview video coding and we will also carry out further investigations for applying our solution to more advanced FEC codes, such as turbo codecs [34], LDPC codes, BICM, BICMID, TCM aided TTCM schemes.

\section{REFERENCES}

[1] L. Hanzo, P. Cherriman, and J. Streit, Video Compression and Communications: From Basics to H.261, H.263, H.264, MPEG2, MPEG4 for DVB and HSDPA-Style Adaptive Turbo-Transceivers. New York: John Wiley, 2007. 
[2] T. Zhang and Y. Xu, "Unequal packet loss protection for layered video transmission," IEEE Transactions on Broadcasting, vol. 45, pp. 243 252, June 1999.

[3] H. Imaizumi and A. Luthra, Three-Dimensional Television, Video and Display Technologies, ch. MPEG-2 Multiview Profile, pp. 169-181. Berlin, Heidelberg, and New York: Springer Verlag, 2002.

[4] H. Schwarz, D. Marpe, and T. Wiegand, "Overview of the scalable video coding extension of the H.264/AVC standard," IEEE Transactions on Circuits and Systems for Video Technology, vol. 17, pp. 1103-1120, September 2007

[5] Joint Video Team (JVT) of ISO/IEC MPEG and ITU-T VCEG, ITUT Rec. H.264/ISO/IEC 14496-10 AVC: Advanced Video Coding for Generic Audiovisual Services, March 2010

[6] A. Vetro, T. Wiegand, and G. Sullivan, "Overview of the stereo and multiview video coding extensions of the H.264/MPEG-4 AVC standard,' Proceedings of the IEEE, vol. 99, pp. 626-642, April 2011.

[7] F. Yang, Q. Zhang, W. Zhu, and Y.-Q. Zhang, "End-to-end TCP-friendly streaming protocol and bit allocation for scalable video over wireless Internet," IEEE Journal on Selected Areas in Communications, vol. 22 pp. 777-790, May 2004.

[8] B. Masnick and J. Wolf, "On linear unequal error protection codes," IEEE Transactions on Information Theory, vol. 13, pp. 600-607, October 1967.

[9] T. Brüggen and P. Vary, "Unequal error protection by modulation with unequal power allocation," IEEE Communications Letters, vol. 9 pp. 484-486, June 2005.

[10] V. Pavlushkov, R. Johannesson, and V. Zyablov, "Unequal error protection for convolutional codes," IEEE Transactions on Information Theory, vol. 52, pp. 700-708, February 2006.

[11] J. Hagenauer, "Rate-compatible puncture convolutional codes (RCPC) and their application," IEEE Transactions on Communications, vol. 36, pp. 389-400, April 1988.

[12] N. Rahnavard and F. Fekri, "New results on unequal error protection using LDPC codes," IEEE Communications Letters, vol. 10, pp. 43-45, January 2006.

[13] V. Kumar and O. Milenkovic, "On unequal error protection LDPC codes based on Plotkin-type constructions," IEEE Transactions on Communications, vol. 54, pp. 994-1005, June 2006.

[14] C. Gong, G. Yue, and X. Wang, "Message-wise unequal error protection based on low-density parity-check codes," IEEE Transactions on Communications, vol. 59, pp. 1019-1030, April 2011.

[15] N. Rahnavard, H. Pishro-Nik, and F. Fekri, "Unequal error protection using partially regular LDPC codes," IEEE Transactions on Communications, vol. 55, pp. 387-391, March 2007.

[16] F. Marx and J. Farah, "A novel approach to achieve unequal error protection for video transmission over 3G wireless networks," Signal Processing: Image Communication, vol. 19, no. 4, pp. 313-323, 2004.

[17] S. X. Ng, J. Y. Chung, and L. Hanzo, "Turbo-detected unequal protection MPEG-4 wireless video telephony using multi-level coding, trellis coded modulation and space-time trellis coding," IEE Proceedings Communications, vol. 152, pp. 1116-1124, December 2005.

[18] H. Wang, F. Zhai, Y. Eisenberg, and A. Katsaggelos, "Cost-distortion optimized unequal error protection for object-based video communications," IEEE Transactions on Circuits and Systems for Video Technology, vol. 15, pp. 1505-1516, December 2005.

[19] M. Aydinlik and M. Salehi, "Turbo coded modulation for unequal error protection," IEEE Transactions on Communications, vol. 56, pp. 555564, April 2008

[20] H. Ha and C. Yim, "Layer-weighted unequal error protection for scalable video coding extension of H.264/AVC," IEEE Transactions on Consumer Electronics, vol. 54, pp. 736-744, May 2008.

[21] Y. C. Chang, S. W. Lee, and R. Komiya, "A fast forward error correction allocation algorithm for unequal error protection of video transmission over wireless channels," IEEE Transactions on Consumer Electronics, vol. 54, pp. 1066-1073, August 2008.

[22] Y. C. Chang, S. W. Lee, and R. Komiya, "A low complexity hierarchical QAM symbol bits allocation algorithm for unequal error protection of wireless video transmission," IEEE Transactions on Consumer Electronics, vol. 55, pp. 1089-1097, August 2009.

[23] D. Sejdinović, D. Vukobratović, A. Doufexi, V. Šenk, and R. Piechocki, "Expanding window fountain codes for unequal error protection," IEEE Transactions on Communications, vol. 57, no. 9, pp. 2510-2516, 2009.

[24] D. Vukobratović, V. Stanković, D. Sejdinović, L. Stanković, and Z. Xiong, "Scalable video multicast using expanding window fountain codes," IEEE Transactions on Multimedia, vol. 11, no. 6, pp. 1094-1104, 2009.
[25] E. Maani and A. Katsaggelos, "Unequal error protection for robust streaming of scalable video over packet lossy networks," IEEE Transactions on Circuits and Systems for Video Technology, vol. 20, pp. 407416, March 2010.

[26] S. Ahmad, R. Hamzaoui, and M. Al-Akaidi, "Unequal error protection using fountain codes with applications to video communication," IEEE Transactions on Multimedia, vol. 13, pp. 92-101, February 2011.

[27] K. Nguyen, T. Nguyen, and S.-C. Cheung, "Video streaming with network coding," Journal of Signal Processing Systems, vol. 59, pp. 319333, June 2010.

[28] C. Hellge, D. Gomez-Barquero, T. Schierl, and T. Wiegand, "Layeraware forward error correction for mobile broadcast of layered media,' IEEE Transactions on Multimedia, vol. 13, pp. 551-562, June 2011.

[29] Nasruminallah and L. Hanzo, "Near-capacity H.264 multimedia communications using iterative joint source-channel decoding," IEEE Communications Surveys and Tutorials, vol. 14, pp. 538-564, Second Quarter 2012.

[30] M. Halloush and H. Radha, "Network coding with multi-generation mixing: A generalized framework for practical network coding," IEEE Transactions on Wireless Communications, vol. 10, no. 2, pp. 466-473, 2011.

[31] Y. Huo, M. El-Hajjar, and L. Hanzo, "Inter-layer FEC aided unequal error protection for multilayer video transmission in mobile TV," IEEE Transactions on Circuits and Systems for Video Technology, vol. 23, no. 9, pp. 1622-1634, 2013.

[32] D. Wu, Y. T. Hou, and Y.-Q. Zhang, "Transporting real-time video over the Internet: challenges and approaches," Proceedings of the IEEE, vol. 88 , no. 12 , pp. $1855-1877,2000$.

[33] T. Stockhammer, M. Hannuksela, and T. Wiegand, "H.264/AVC in wireless environments," IEEE Transactions on Circuits and Systems for Video Technology, vol. 13, no. 7, pp. 657-673, 2003

[34] L. Hanzo, T. Liew, B. Yeap, and R. Tee, Turbo Coding, Turbo Equalisation and Space-Time Coding. New York: John Wiley, 2011.

[35] T. Stockhammer, A. Shokrollahi, M. Watson, M. Luby, and T. Gasiba, "Application Layer Forward Error Correction for Mobile Multimedia Broadcasting," in Handbook of Mobile Broadcasting: DVB-H, DMB ISDB-T and Media FLO (B. Furhet and S. Ahson, eds.), pp. 239-280, Boca Raton, FL: CRC Press, 2008.

[36] C. Hellge, T. Schierl, and T. Wiegand, "Multidimensional layered forward error correction using rateless codes," in IEEE International Conference on Communications, (Beijing, China), pp. 480-484, May 2008.

[37] S. ten Brink, G. Kramer, and A. Ashikhmin, "Design of low-density parity-check codes for modulation and detection," IEEE Transactions on Communications, vol. 52, pp. 670-678, April 2004

[38] J. Hagenauer, E. Offer, and L. Papke, "Iterative decoding of binary block and convolutional codes," IEEE Transactions on Information Theory, vol. 42, pp. 429-445, March 1996.

[39] J. Chen, A. Dholakia, E. Eleftheriou, M. Fossorier, and X.-Y. Hu, "Reduced-complexity decoding of LDPC codes," IEEE Transactions on Communications, vol. 53, pp. 1288-1299, August 2005.

[40] C. Berrou, A. Glavieux, and P. Thitimajshima, "Near Shannon limit error-correcting coding and decoding: Turbo codes," in IEEE International Conference on Communications, (Geneva, Switzerland), pp. 1064-1070, May 1993

[41] S. ten Brink, "Convergence behavior of iteratively decoded parallel concatenated codes," IEEE Transactions on Communications, vol. 49, pp. 1727-1737, October 2001.

[42] R. Maunder and L. Hanzo, "Extrinsic information transfer analysis and design of block-based intermediate codes," IEEE Transactions on Vehicular Technology, vol. 60, pp. 762-770, March 2011.

[43] R. Khalili and K. Salamatian, "A new analytic approach to evaluation of packet error rate in wireless networks," in Communication Networks and Services Research Conference, (Halifax, Nova Scotia, Canada), pp. 333 338, May 2005.

[44] X. Yang, C. Zhu, Z. Li, X. Lin, G. Feng, S. Wu, and N. Ling, "Unequal loss protection for robust transmission of motion compensated video over the Internet," Signal Processing: Image Communication, vol. 18 no. 3, pp. 157-167, 2003.

[45] M. Clerc and J. Kennedy, "The particle swarm - explosion, stability, and convergence in a multidimensional complex space," IEEE Transactions on Evolutionary Computation, vol. 6, no. 1, pp. 58-73, 2002.

[46] D. Schonfeld and N. Bouaynaya, "A new method for multidimensional optimization and its application in image and video processing," IEEE Signal Processing Letters, vol. 13, no. 8, pp. 485-488, 2006. 
[47] Z.-H. Zhan, J. Zhang, Y. Li, and H.-H. Chung, "Adaptive particle swarm optimization," IEEE Transactions on Systems, Man, and Cybernetics, Part B: Cybernetics, vol. 39, no. 6, pp. 1362-1381, 2009.

[48] A. Detti, G. Bianchi, C. Pisa, F. Proto, P. Loreti, W. Kellerer, S. Thakol sri, and J. Widmer, "SVEF: an open-source experimental evaluation framework for H.264 scalable video streaming," in IEEE Symposium on Computers and Communications, ISCC, (Sousse, Tunisia), pp. 3641, July 2009

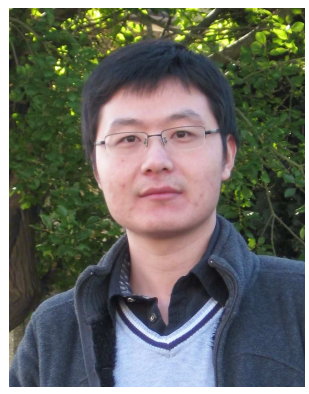

Yongkai Huo received the B.Eng. degree with distinction in computer science and technology from Hefei University of Technology, Hefei, China, in 2006 and the M.Eng. degree in computer software and theory from University of Science and Technology of China, Hefei, China, in 2009. He is currently working toward the Ph.D. degree with the Communications, Signal Processing and Control Group, School of Electronics and Computer Science, University of Southampton, Southampton, U.K. He received a scholarship under the China-U.K. Scholarships for Excellence Programme. His research interests include distributed video coding, multiview video coding, robust wireless video streaming and joint source-channel decoding.

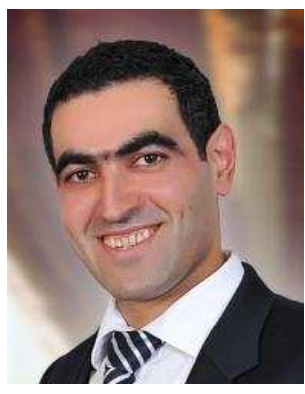

Mohammed El-Hajjar is a lecturer in the Electronics and Computer Science in the University of Southampton. He received his BEng degree in Electrical Engineering from the American University of Beirut, Lebanon in 2004. He then received an MSc in Radio Frequency Communication Systems and $\mathrm{PhD}$ in Wireless Communications both from the University of Southampton, UK in 2005 and 2008, respectively. Following the $\mathrm{PhD}$, he joined Imagination Technologies as a research engineer, where he worked on designing and developing the BICM peripherals in Imagination's multistandard communications platform, which resulted in several patent applications. In January 2012, he joined the Electronics and Computer Science in the University of Southampton as a lecturer in the Communications, Signal Processing and Control research group. He is the recipient of several academic awards and has published a Wiley-IEEE book and in excess of 30 journal and international conference papers. His research interests are mainly in the development of intelligent communications systems for the Internet of Things including massive MIMO systems for mm-wave communications, cooperative communications and Radio over fibre systems.

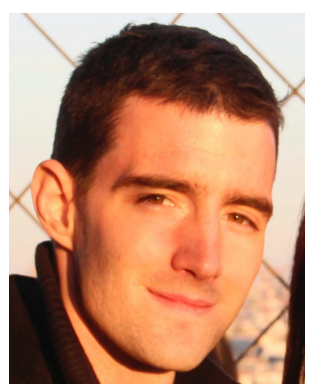

Robert G. Maunder has studied with Electronics and Computer Science, University of Southampton, UK, since October 2000. He was awarded a first class honors BEng in Electronic Engineering in July 2003, as well as a $\mathrm{PhD}$ in Wireless Communications and a lectureship in December 2007. Rob's research interests include joint source/channel coding, iterative decoding, irregular coding and modulation techniques. He has published a number of IEEE papers in these areas.

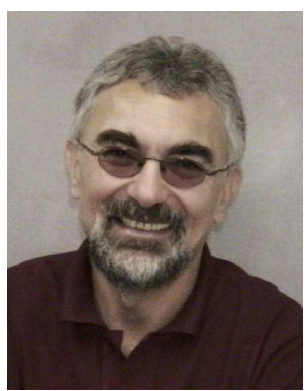

Lajos Hanzo (F' 04) received the degree in electronics in 1976, the Doctorate degree in 1983 , and the D.Sc. degree.

During his 35-year career in telecommunications he has held various research and academic posts in Hungary, Germany and the UK. Since 1986 he has been with the School of Electronics and Computer Science, University of Southampton, UK, where he holds the chair in telecommunications. He has successfully supervised $80 \mathrm{PhD}$ students, co-authored $20 \mathrm{John}$ Wiley/IEEE Press books on mobile radio communications totalling in excess of 10000 pages, published 1300 research entries at IEEE Xplore, acted both as TPC and General Chair of IEEE conferences, presented keynote lectures and has been awarded a number of distinctions. Currently he is directing a 100 -strong academic research team, working on a range of research projects in the field of wireless multimedia communications sponsored by industry, the Engineering and Physical Sciences Research Council (EPSRC) UK, the European IST Programme and the Mobile Virtual Centre of Excellence (VCE), UK. He is an enthusiastic supporter of industrial and academic liaison and he offers a range of industrial courses. He is also a Governor of the IEEE VTS. During 2008 - 2012 he was the Editor-in-Chief of the IEEE Press and since 2009 he has been a Chaired Professor also at Tsinghua University, Beijing. For further information on research in progress and associated publications please refer to http://www-mobile.ecs.soton.ac.uk 